\title{
Nonlinear electrodynamics as a symmetric hyperbolic system
}

\author{
Fernando Abalos, ${ }^{1, *}$ Federico Carrasco, ${ }^{1, \dagger}$ Érico Goulart, ${ }^{2, \ddagger}$ and Oscar Reula ${ }^{1, \S}$ \\ ${ }^{1}$ Facultad de Matemática, Astronomía y Física, Universidad Nacional de Córdoba and IFEG-CONICET, \\ Ciudad Universitaria, X5016LAE Córdoba, Argentina \\ ${ }^{2}$ CAPES Foundation, Ministry of Education, Brasilia, Distrito Federal 70.040-020, Brazil
}

(Received 4 August 2015; published 9 October 2015)

\begin{abstract}
Nonlinear theories generalizing Maxwell's electromagnetism and arising from a Lagrangian formalism have dispersion relations in which propagation planes factor into null planes corresponding to two effective metrics which depend on the pointwise values of the electromagnetic field. These effective Lorentzian metrics share the null (generically two) directions of the electromagnetic field. We show that the theory is symmetric hyperbolic if and only if the cones these metrics give rise to have a nonempty intersection, namely, that there exist families of symmetrizers in the sense of Geroch [26] which are positive definite for all covectors in the interior of the cones intersection. Thus, for these theories, the initial value problem is well posed. We illustrate the power of this approach with several nonlinear models of physical interest such as Born-Infeld, Gauss-Bonnet, and Euler-Heisenberg.
\end{abstract}

\section{INTRODUCTION}

Nonlinear electrodynamics (NLED) is relevant in several areas of physics. In QED, the polarization of the vacuum leads naturally to nonlinear effects (such as the light-light scattering) which are effectively described by a EulerHeisenberg's Lagrangian [1-6] (see also Ref. [7] for a pedagogical review). In some dielectrics and crystals, the interaction between the molecules and external electromagnetic fields can be described by an effective nonlinear theory, which is typically observed at very high light intensities such as those provided by pulsed lasers [8,9]. Possible consequences of NLED have been explored also in cosmology and astrophysics. In particular, it is believed that nonlinearities may play important roles in the description of the dark sector of the Universe [10-15], in the avoidance of singularities (when coupled to Einstein's equations), or in the physics of charged black holes [16-21]. These models have the advantage of using only electromagnetic fields, without invoking yet unobservable scalars or more speculative ideas related to higher dimensions and brane worlds. Finally, the Born-Infeld nonlinear model [22-24] has mathematical connections to string theory, for its Lagrangian appears in relation with the gauge fields on a D-brane (see, for instance, Ref. [25]). In general, NLED theories attract attention because they offer insight into light propagation in the experimental and theoretical studies of relativity.

A key feature to elucidate about the partial differential equations (PDEs) governing NLED is whether they pose an

\footnotetext{
*jfera18@gmail.com

fedecarrasco@gmail.com

*egoulart2@gmail.com

\$reula@famaf.unc.edu.ar
}

initial value formulation. Well posedness is at the roots of physics, for it amounts to the predictability power of the theory, asserting that solutions exist, are unique, and depend continuously on the initial data. The mathematical theory dealing with the initial value formulation is well developed, and for this case it amounts to checking whether the first-order system of quasilinear PDEs is hyperbolic and what the maximal propagation speeds are. Roughly, hyperbolicity is an algebraic property of the principal symbol (the differential operator consisting of the highest derivative order terms of the PDE), which is essential to prove local well posedness of the noncharacteristic Cauchy problem. In theories like NLED, the analysis of hyperbolicity needs, however, a careful manipulation. Because constraints are present, the evolution equations are not uniquely defined for one can add to any one of them constraint terms and obtain another equivalent evolution system. The distinction of an evolution system over the others generally implies a choice of $3+1$ decomposition of space-time and, as such, breaks covariance.

In this paper, we shall treat the equivalent evolution systems in an equal footing so as to assert the desired hyperbolicity properties keeping covariance as much as we can. The tools to deal with hyperbolicity in this way has been provided by Geroch in Ref. [26]. However, as there are many different notions of hyperbolicity available in the literature (see, e.g., Refs. [27-29]), we now recall some previous results concerning the evolutionary aspects of NLED. In Ref. [30], for instance, Brenier uses the energy density and the Poynting vector as additional unknowns to augment the original $6 \times 6$ Born-Infeld system to a system of $10 \times 10$ hyperbolic conservation laws (for which wellknown results can be directly applied [31-33]). A similar analysis using convex entropies (actually the energy) may be found in Ref. [34], where Serres extends a method 
designed by Dafermos [35] (see also Ref. [36]) to the class of models described by Coleman and Dill [37]. As a result, he shows that the polyconvexity of the energy density implies the local well posedness of the Cauchy problem within smooth functions of class $H^{s}$ with $s>1+d / 2$. Another approach using a $3+1$ splitting was provided by V. Perlick in Ref. [38], assuming that the constitutive equations $\mathcal{F}_{A}(H, F)=0$ can be solved for $\vec{E}$ and $\vec{H}$. Several results that are relevant for the question of whether the evolution equations are hyperbolic, strongly hyperbolic, or symmetric hyperbolic are investigated in detail. It is worth mentioning also the global results by Speck [39]. Using ideas presented by Christodoulou and Klainerman $[40,41]$, he establishes the existence of small-data global solutions to the Born-Infeld system on the Minkowski space background in $1+3$ dimensions. Roughly, he concludes that, if the initial data for the Born-Infeld equations are sufficiently small as measured by a weighted Sobolev norm, then these data launch a unique classical solution to the equations existing in all of Minkowski space. Furthermore, he shows that these solutions decay at exactly the same rates as solutions to the linear Maxwell system.

Here, we adopt a different strategy. Working within Geroch's geometrical formalism, we find the most general hyperbolizations NLED theories admit and show that they are parametrized by an auxiliary vector field $t^{q}(x)$ (as is also the case for linear electrodynamics). This construction allows us to find necessary and sufficient conditions theories and fields must satisfy in order to have a wellposed initial value formulation. It happens that such a condition translates very nicely into geometrical terms: the system is symmetric hyperbolic, if and only if the two cones arising from the dispersion relations (or, conversely, the characteristic surfaces) of the given NLED theory have a nonempty intersection. This constitutes one of the main results of the present article. Our construction also allows us to find the causality cone (the maximal propagation speeds) each hyperbolization has. These cones could be, in principle, different from the physical cones as defined by the dispersion relations, for the latter ones use all equations, including the constraints, to assert the propagation velocities of plane waves, while the hyperbolization cones use only the corresponding evolution equations. We find that, nevertheless, they coincide.

\section{A. A warning on terminology}

In the present context, cones appear in several related disguises. First, we have the familiar cones arising in Lorentzian geometry, namely, those arising from the set of all timelike vectors $\left\{v \in T_{p} \mathbf{M} \mid g_{a b} v^{a} v^{b}>0\right\}$, which splits into two disjoint sets, the "future" and "past" propagation cones. We shall refer to them as the cones of a given metric. Second, now allowing for more general symmetrichyperbolic systems, the cones that appear as the set of covectors which make positive definite a certain symmetric hyperbolizer, $C_{H}^{*}:=\left\{n_{a} \in T_{p}^{*} \mathbf{M} \mid H_{\alpha \beta}^{a} n_{a}>0\right\}$. These cones are clearly open and convex, for if $n_{a}$ and $n_{a}^{\prime}$ belong to $C_{H}^{*}$, so does $\lambda_{1} n_{a}+\lambda_{2} n_{a}^{\prime}$ for all positive $\lambda_{1}, \lambda_{2}$, since the sum of positive bilinear forms gives another positive bilinear form. They represent planes on the tangent space. Once a plane is found within $C_{H}^{*}$, the others are found tilting it until $H_{\alpha \beta}^{a} n_{a}$ gets a kernel. Each one of these planes represents a plane wave perturbation solution to the underlying equations out of which the symmetrizer was built upon. Third, we can construct the cocones, duals to the previous ones; that is, given a cone, $C \in V$, we can define a cone in the dual space, $C^{\prime}:=\left\{\sigma \in V^{\prime} \mid \sigma(v) \geq 0, \quad \forall v \in C\right\}$, and this is clearly also a convex close cone. The Cocones dual to the cones defined from hyperbolizers, are called the propagation cones, for they determine the directions along which perturbations propagate. Notice that the cocones of a given metric cone are precisely those covectors obtained by lowering with the metric the indices of the all the vectors forming the cones, so the distinction is lost. However, since in this work there appear several Lorentzian metrics, it is best to keep cones and cocones as separate geometrical entities.

This article is organized as follows. In Sec. II, we introduce NLED equations and point out some of their basic structural properties. We then review some important results concerning characteristic surfaces and geometrical aspects these theories exhibit. In Sec. III, an intrinsic geometrical formulation of PDEs due to Geroch is introduced, and the notion of symmetric hyperbolicity is presented. We then investigate the algebraic core of the NLED equations and find the most general hyperbolizations these theories allow. Our main results are presented in this section in the form of two theorems, together with (we hope) comprehensive descriptions of the key ideas behind the proofs. In Sec. IV, we provide the reader with the detailed steps to prove the theorems, and Sec. V deals with the constraints. Finally, Sec. VI explores our results for some particular realizations of the Lagrangian.

\section{GENERAL REMARKS}

\section{A. Lagrangians and equations of motion}

Write $(\mathbf{M}, g)$ for a $(1+3)$-dimensional space-time, with signature convention $(+,-,-,-)$, and $F_{a b}$ for the electromagnetic 2-form. Let

$$
F:=F^{a b} F_{a b}=2\left(H^{2}-E^{2}\right) \quad G:=F^{a b} F_{a b}^{*}=4 \vec{E} \cdot \vec{H}
$$

denote the field invariants, where $F_{a b}^{*}=\frac{1}{2} \eta_{a b c d} F^{c d}$ stands for the dual, $\eta_{a b c d}$ stands for the completely antisymmetric Levi-Civitá tensor, and $(\vec{E}, \vec{H})$ stands for the electromagnetic fields. We consider nonlinear models of electrodynamics in vacuum provided by the action 


$$
S=\int \mathcal{L}(F, G) \sqrt{-g} d^{4} x,
$$

where the Lagrangian density $\mathcal{L}(F, G)$ is an arbitrary smooth function of the invariants and $g:=\operatorname{det}\left(g_{a b}\right)$. The first-order, quasilinear, equations read as

$$
\nabla_{a}\left(\mathcal{L}_{F} F^{a b}+\mathcal{L}_{G} F^{*}\right)=0, \quad \nabla_{[a} F_{b c]}=0,
$$

with $\nabla$ denoting covariant derivative and $\mathcal{L}_{X}:=\frac{\partial \mathcal{L}}{\partial X}$, for conciseness. Here, the lhs system is obtained via the variational principle, while the rhs system is assumed from the very beginning, thus guaranteeing the existence of a 4-potential such that $F_{a b}=\nabla_{[a} A_{b]}$. We define also, for future convenience, the quantities

$\xi_{1}:=2 \mathcal{L}_{F F} / \mathcal{L}_{F}, \quad \xi_{2}:=2 \mathcal{L}_{F G} / \mathcal{L}_{F}, \quad \xi_{3}:=2 \mathcal{L}_{G G} / \mathcal{L}_{F}$

Remark 1: In Maxwell's electrodynamics, there exists a particular gauge which considerably simplifies the second-order equations for $A_{a}(x)$. Unfortunately, this is not the case for more general nonlinear theories, and it will be convenient to focus our analysis on the first-order equations (3) only. Also, currents $j_{a}(x)$ are irrelevant for the question of whether the initial value problem is well posed. Therefore, they will play no role in our further discussion.

\section{B. Dispersion relations, characteristics, and effective metrics}

It is well known that high-frequency perturbations about a smooth background solution of Eqs. (3) are controlled by two effective metrics. ${ }^{1}$ The core of this result was first presented by Boillat [44] and Plebanski [45] in the early 1970s using Hadamard's method of discontinuities [46]. More recently, Obukhov and Rubilar [47] noticed that quasilinear PDE's of the form (3) are particular instances of electrodynamics inside media described by general nonlinear constitutive laws. As a consequence, they showed that, if $\Sigma$ is a characteristic hypersurface described by $f\left(x^{a}\right)=$ const, the wave normals $k_{m}:=\partial_{m} f$ are given by the vanishing sets of a fourth-order multivariate polynomial in the cotangent bundle $T^{*} \mathbf{M}$,

$$
\mathcal{P}^{*}(x, k):=\mathfrak{G}^{a b c d}(x) k_{a} k_{b} k_{c} k_{d}=0 .
$$

Here, $\mathfrak{G S}^{a b c d}(x)$ is a completely symmetric quantity (35 independent components) depending implicitly on the background solution. Physically, Eq. (5) plays the role

\footnotetext{
${ }^{1}$ See Refs. [42] and [43] for a wealth of details in the context of analog models of gravity.
}

of a dispersion relation for the linearized waves and gives rise to some sort of covariant Fresnel equation [48,49].

For the class of nonlinear Lagrangian models provided by (2), a remarkable property holds due to algebraic conditions: the multivariate polynomial (5) always reduces to the simpler form

$$
\mathcal{P}^{*}(x, k)=a k^{4}+Q k^{2} l^{2}+R l^{4},
$$

with $k^{2}=g^{a b} k_{a} k_{b}, l^{2}=F^{a}{ }_{c} F^{b c} k_{a} k_{b}$, for conciseness, and

$$
\begin{gathered}
a:=\left(1+\xi_{2} G-\xi_{3} F-R G^{2} / 16\right), \\
Q:=2\left(\xi_{1}+\xi_{3}-R F / 4\right), \\
R:=4\left(\xi_{1} \xi_{3}-\xi_{2}^{2}\right) .
\end{gathered}
$$

A closer inspection of (6) reveals that the quartic Fresnel surface of the wave normals factorizes to the product of two second-order surfaces, given in terms of the quadratic forms

$$
\mathfrak{g}_{1}^{a b}(x) k_{a} k_{b}=0, \quad \mathfrak{g}_{2}^{a b}(x) k_{a} k_{b}=0,
$$

with the reciprocal effective metrics given by

$$
\begin{gathered}
\mathfrak{g}_{1}^{a b}:=a g^{a b}+b_{1} F^{a}{ }_{c} F^{b c}, \\
\mathfrak{g}_{2}^{a b}:=g^{a b}+\left(b_{2} / a\right) F_{c}^{a} F^{b c},
\end{gathered}
$$

according to the following definitions ${ }^{2}$ :

$b_{1}:=\frac{Q+\sqrt{\Delta}}{2}, \quad b_{2}:=\frac{Q-\sqrt{\Delta}}{2}, \quad \Delta:=Q^{2}-4 a R$.

As stressed by Boillat [44], the roots always exist since the discriminant is actually a sum of squares, i.e., $\Delta=$ $4\left(N_{1}^{2}+N_{2}^{2}\right)$ with

$$
N_{1}:=\left(\xi_{1}-\xi_{3}\right)-R F / 4, \quad N_{2}:=2 \xi_{2}-R G / 4 .
$$

Obukhov and Rubilar proceed by showing us that $\mathfrak{g}_{1}^{a b}$ and $\mathfrak{g}_{2}^{a b}$ are Lorentzian whenever the background spacetime metric is Lorentzian. Their results are in qualitative agreement with previous results by Novello et al. [50,51], wherein the rays spanning the characteristic surfaces are often described in terms of effective null geodesics. Therefore, in what follows, we shall assume that the

\footnotetext{
${ }^{2}$ If $a \rightarrow 0$ but $b_{1} \neq 0$, the left-hand side of (12) is still finite since $b_{2} / a=R / b_{1}$. When $a \rightarrow 0$ and $b_{1} \rightarrow 0$ simultaneously, we can, using the conformal freedom, redefine the metrics as $\tilde{\mathfrak{g}}_{1}^{a b}:=\left(1 / b_{1}\right) \mathfrak{g}_{1}^{a b}, \tilde{\mathfrak{g}}_{2}^{a b}:=b_{1} \tilde{g}_{2}^{a b}$. The results we shall obtain do not depend on this reparametrization.
} 
effective metrics are always Lorentzian. ${ }^{3}$ For the covariant components of the latter, we write $\mathfrak{g}_{a b}^{1}$ and $\mathfrak{g}_{a b}^{2}$. They are such that $\mathfrak{g}_{1}^{a c} \mathfrak{g}_{c b}^{1}=\delta^{a}{ }_{b}$ and $\mathfrak{g}_{2}^{a c} \mathfrak{g}_{c b}^{2}=\delta^{a}{ }_{b}$.

\section{Geometrical structure: The cones}

The effective metrics are defined up to a conformal transformation. However, they have an intrinsically geometric property which does not depend on conformal redefinitions, namely, their cones. ${ }^{4}$ We will denote by $C_{\mathfrak{g}_{i}}$ the set of all timelike (future-directed) vectors with respect to the metrics $\mathfrak{g}_{i}{ }^{5}$, and $C_{\mathfrak{g}_{i}}^{*}:=$ $\operatorname{int}\left(\left\{n_{a} \in T_{p}^{*} \mathbf{M} \mid n_{a} v^{a}>0, \quad \forall v^{a} \in C_{\mathbf{g}_{i}}\right\}\right)^{6} \quad$ their dual cocones. We study here some geometrical relations among these cones, since they will play an important role in the description of hyperbolicity. To do that, we distinguish between two different cases, according to the nature of the electromagnetic field $F_{a b}:$ i) nondegenerate fields $\left(F^{2}+G^{2} \neq 0\right)$ and ii) degenerate fields $(F=G=0)$. As these cases have different geometric interpretations, we shall treat them separately (see also Ref. [52]).

\section{Nondegenerate $F_{a b}$}

A nondegenerate 2-form at a point $p$ has (at that point) two null eigenvectors $k^{a}$ and $l^{a}$ (see Appendix A for further details). The directions of these vectors are called the principal null directions (PND') [53-55] and will play a key role in our description.

Remark 2: $\quad k^{a}$ and $l^{a}$ are null with respect to the three metrics, that is, the background and the effective metrics. We shall say henceforth that the closures of all cones share these principal null directions. The plane spanned by $k^{a}$ and $l^{a}$ forms the essential geometrical structure of the system.

The PNDs help us to construct a frame which simplifies calculations considerably. In this frame, $\vec{E} \| \vec{H}, g_{a b}$ reduces to $\eta_{a b}$, and one obtains the quadratic forms

$$
\begin{aligned}
\mathfrak{g}_{a b}^{i} t^{a} t^{b} & :=\alpha_{i}^{-1}\left(t_{0}^{2}-t_{3}^{2}\right)-\beta_{i}^{-1}\left(t_{1}^{2}+t_{2}^{2}\right) \\
\mathfrak{g}_{i}^{a b} n_{a} n_{b} & :=\alpha_{i}\left(n_{0}^{2}-n_{3}^{2}\right)-\beta_{i}\left(n_{1}^{2}+n_{2}^{2}\right)
\end{aligned}
$$

with $i=1,2$ and

\footnotetext{
${ }^{3} \mathrm{~A}$ degenerate metric does not give rise to a cone according to our definition. Therefore, we analyze them separately in Appendix B, where we conclude those systems are not symmetric hyperbolic.

${ }^{4}$ By a cone, we mean the interior of a proper cone. Recall that a proper cone $C$ is a subset of a vector space $V$ such that $(\alpha \mathbf{u}+\beta \mathbf{v}) \in V \forall \mathbf{u}, \mathbf{v} \in V \forall \alpha, \beta>0$, and $\bar{C} \cap-\bar{C}=\emptyset$.

${ }^{5}$ For symmetric hyperbolic systems once a symmetrizer is given we will adopt for all effective metrics the convention that the future cones are those with a nonvanishing intersection with the corresponding propagation cone. In the case the system is not symmetric hyperbolic we shall take into account all cones, two for each metric, and refer to all of them as cones.

${ }^{6} \mathrm{We}$ choose the interior, so as to make the dual cones open, and thus put them in an equal footing with the cones $C_{\mathfrak{g}_{i}}$.
}

$$
\begin{aligned}
& \alpha_{1}:=a+b_{1} \frac{1}{4}\left(F-\sqrt{F^{2}+G^{2}}\right) \\
& \beta_{1}:=a+b_{1} \frac{1}{4}\left(F+\sqrt{F^{2}+G^{2}}\right) \\
& \alpha_{2}:=1+\frac{b_{2}}{a} \frac{1}{4}\left(F-\sqrt{F^{2}+G^{2}}\right) \\
& \beta_{2}:=1+\frac{b_{2}}{a} \frac{1}{4}\left(F+\sqrt{F^{2}+G^{2}}\right) .
\end{aligned}
$$

Note that the quantities involved in (16) are covariantly defined; i.e., they are functions of the invariants $F$ and $G$.

A closer inspection of (15) reveals that the effective cones are characterized by the signs of the coefficients (16). We introduce to this matter two useful quantities

$$
\Omega_{i}:=\alpha_{i} \beta_{i} \quad(\text { for } i=1,2)
$$

and present a table illustrating all possible sign combinations:

\begin{tabular}{lcccccc}
\hline & $\begin{array}{c}\text { Sign } \\
\left(\alpha_{i}\right)\end{array}$ & $\begin{array}{c}\text { Sign } \\
\left(\beta_{i}\right)\end{array}$ & $\begin{array}{c}\text { Signature } \\
\text { of } \mathfrak{g}_{i}\end{array}$ & $\begin{array}{c}\text { Timelike } \\
\text { vector }\end{array}$ & $\mathfrak{g}_{a b}^{i} t^{a} t^{b}$ & $\begin{array}{c}\text { Sign } \\
\left(\Omega_{i}\right)\end{array}$ \\
\hline i) & + & + & $(+,-,-,-)$ & $t^{a}=(1,0,0,0)$ & $\alpha_{1}^{-1}>0$ & + \\
ii) & - & - & $(-,+,+,+)$ & $t^{a}=(1,0,0,0)$ & $\alpha_{1}^{-1}<0$ & + \\
iii $)$ & + & - & $(+,+,+,-)$ & $t^{a}=(0,0,0,1)$ & $-\alpha_{1}^{-1}<0$ & - \\
iv) & - & + & $(-,-,-,+)$ & $t^{a}=(0,0,0,1)$ & $-\alpha_{1}^{-1}>0$ & - \\
\hline
\end{tabular}

Note that the signatures of the effective metrics are not determined by their Lorentzian character. In particular, the norm of timelike vectors depends on the background field and does not have a preferred sign ${ }^{7}$ The cones, on the other hand, are clearly independent on how these signatures turn out, and the same will apply to all our future results, as we shall see later.

Proposition 1: (i) If $\Omega_{i}>0$, then $C_{\mathbf{g}_{i}} \cap C_{\eta} \neq \emptyset$. [where $\left.\eta^{a b}:=\operatorname{Diag}(1,-1,-1,-1)\right]$. (ii) If $\Omega_{i}<0$, then $C_{\mathfrak{g}_{i}} \cap C_{\sigma} \neq \emptyset$. [where $\sigma^{a b}:=\operatorname{Diag}(-1,-1,-1,1)$ ].

Therefore, there are only three qualitatively different configurations for the effective cones in $T_{p} \mathbf{M}$ : the three cones intersect [Fig. 1(a)]; the two effective cones intersect each other, but do not intersect the background cone [Fig. 1(c)]; or they do not intersect [Fig. 1(b)]. Moreover, when the effective cones do intersect each other, it turns out that it is always possible to single one as being included inside the other. Thus, the following holds:

Proposition 2: Whenever the effective cones intersect each other, i.e., $C_{\mathfrak{g}_{1}} \cap C_{\mathfrak{g}_{2}} \neq \emptyset$, then

(i) $C_{\mathfrak{g}_{1}}^{*} \subseteq C_{\mathfrak{g}_{2}}^{*}$

(ii) $C_{\mathfrak{g}_{2}} \subseteq C_{\mathfrak{g}_{1}}$

\footnotetext{
${ }^{7}$ One could in principle redefine the metrics to fix both signatures in accordance with the convention chosen for the background metric, but these redefinitions are going to depend generically on the background fields.
} 


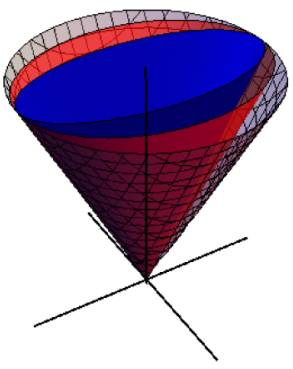

(a)

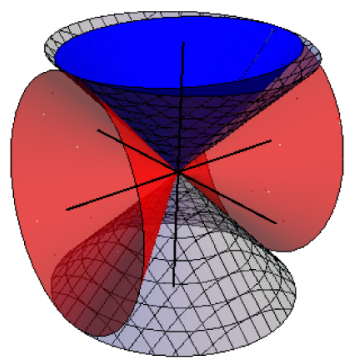

(b)

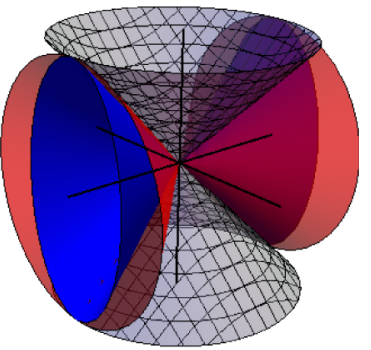

(c)

FIG. 1 (color online). Nondegenerate case. Possible configurations for the null surfaces of the metrics: $\mathfrak{g}_{a b}^{1}$ (red), $\mathfrak{g}_{a b}^{2}$ (blue), and background metric (meshed gray). (a) $\Omega_{1}>0$ and $\Omega_{2}>0$, (b) $\Omega_{1}>0$ and $\Omega_{2}<0$, (c) $\Omega_{1}<0$ and $\Omega_{2}<0$.

Proof.-We begin our proof with the following important observation:

Lemma 1: The following inequalities appear as algebraic consequences of the definitions:

$$
\alpha_{1} \beta_{2} \leq 1 \leq \alpha_{2} \beta_{1} .
$$

Indeed, starting from the full expressions,

$$
\begin{aligned}
& \alpha_{1} \beta_{2}=1+\frac{1}{2}\left(\left(F N_{1}+G N_{2}\right)-\sqrt{N_{1}^{2}+N_{2}^{2}} \sqrt{F^{2}+G^{2}}\right) \\
& \alpha_{2} \beta_{1}=1+\frac{1}{2}\left(\left(F N_{1}+G N_{2}\right)+\sqrt{N_{1}^{2}+N_{2}^{2}} \sqrt{F^{2}+G^{2}}\right),
\end{aligned}
$$

and the trivial inequality $\alpha_{2} \beta_{1} \geq \alpha_{1} \beta_{2}$, one obtains, after some simple manipulations,

$$
-\left(1-\alpha_{1} \beta_{2}\right)\left(1-\alpha_{2} \beta_{1}\right)=\frac{1}{4}\left(G N_{1}-F N_{2}\right)^{2} \geq 0
$$

The positivity of the left-hand side, together with condition $\alpha_{2} \beta_{1} \geq \alpha_{1} \beta_{2}$, forces the inequalities of the lemma to hold.

We now define two auxiliary quantities which capture the notion of how much the given cone (or cocone) opens in any direction orthogonal to the PNDs plane in this particular frame,

$$
\gamma_{i}:=\sqrt{\left|\alpha_{i} / \beta_{i}\right|} .
$$

Despite the fact that this is a coordinate-dependent notion, it allows us to compare the cones and tell which of them is included in the other. The latter statement has a welldefined geometrical meaning and directly extrapolates to any other frame. The argument goes as follows. The assumption $C_{\mathfrak{g}_{1}} \cap C_{\mathfrak{g}_{2}} \neq \emptyset$ translates into

$$
\begin{aligned}
\operatorname{sign}\left(\Omega_{1}\right) & =\operatorname{sign}\left(\Omega_{2}\right) \quad \text { or equivalently } \\
0<\Omega_{1} \Omega_{2} & =\alpha_{1} \beta_{2} \alpha_{2} \beta_{1},
\end{aligned}
$$

which, together with (18), implies $\alpha_{1} \beta_{2}>0$ and, consequently, $\gamma_{1} \leq \gamma_{2}$. Now, suppose $\Omega_{1}>0$ and $\Omega_{2}>0$, and consider a continuous transition from a timelike to a spacelike covector (with respect to the effective metrics) parametrized by $x \in[0,1]$ in the form

$$
n_{a}(x)=(1-x)(1,0,0,0)+x(0, \cos \phi, \sin \phi, 0)
$$

with $\phi \in[0,2 \pi)$. The idea is to find $x_{1}$ and $x_{2}$ such that the covector becomes null, i.e, $\mathfrak{g}_{i}^{a b} n_{a}\left(x_{i}\right) n_{b}\left(x_{i}\right)=0(i=1,2)$. The solutions are given by

$$
x_{i}=\frac{1}{1+\gamma_{i}^{-1}},
$$

which determines $x_{1} \leq x_{2}$. Conversely, assuming $\Omega_{1}<0$ and $\Omega_{2}<0$, we consider the family

$$
n_{a}(x)=(1-x)(0,0,0,1)+x(0, \cos \phi, \sin \phi, 0)
$$

and get precisely the same answer, i.e., $x_{1} \leq x_{2}$. This allows us to conclude that $C_{\mathfrak{g}_{1}}^{*} \subseteq C_{\mathfrak{g}_{2}}^{*}$ as claimed in (i). To prove (ii), we basically apply the same strategy. Instead of using the reciprocal effective metrics, we now look at the covariant objects $\mathfrak{g}_{a b}^{i}$, and the relevant quantities become $\gamma_{i}^{*}:=\gamma_{i}^{-1}$. One then concludes that our previous inequality is inverted, i.e., $x_{1} \geq x_{2}$. The latter directly leads us to the desired result (ii).

\section{Degenerate $\boldsymbol{F}_{a b}$}

For a degenerate 2-form, the two null directions collapse into a single one (see Fig. 2). Roughly, this means that either the cones do not intersect each other or one of them is included in the other. ${ }^{8}$ Again, we refer the reader to Appendix A for more details on frames. In particular, one can reduce the metrics and inverses to

$$
\begin{aligned}
\mathfrak{g}_{a b}^{i} t^{a} t^{b} & =\left(t_{0}^{2}-t_{3}^{2}\right)+\varepsilon^{4} b_{i}\left(t_{0}-t_{3}\right)^{2}-\left(t_{1}^{2}+t_{2}^{2}\right) \\
\mathfrak{g}_{i}^{a b} n_{a} n_{b} & =\left(n_{0}^{2}-n_{3}^{2}\right)-\varepsilon^{4} b_{i}\left(n_{0}+n_{3}\right)^{2}-\left(n_{1}^{2}+n_{2}^{2}\right)
\end{aligned}
$$

\footnotetext{
${ }^{8}$ This do not exclude the possibility they coincide.
} 


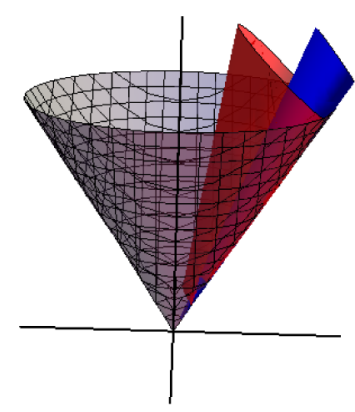

(a)

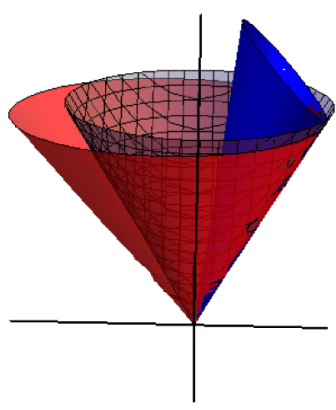

(b)

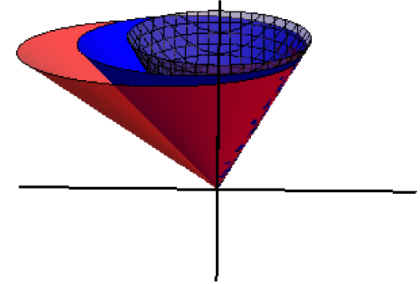

(c)

FIG. 2 (color online). Degenerate case. Possible configurations for the null surfaces of the metrics: $\mathfrak{g}_{a b}^{1}$ (red), $\mathfrak{g}_{a b}^{2}$ (blue), and the background metric (meshed gray). (a) $0<b_{2} \leq b_{1}$, (b) $b_{2}<0<b_{1}$, (c) $b_{2} \leq b_{1}<0$.

with $\varepsilon \neq 0$ a free parameter, a remaining freedom in our frame choice that we will fix in a convenient way later on. Note that when $b_{i}=0$ the effective metric reduces to a background metric.

Proposition 3: For a degenerate $F_{a b}$, the effective cones always intersect among each other, i.e, $C_{\mathfrak{g}_{1}}^{*} \cap C_{\mathfrak{g}_{2}}^{*} \neq \emptyset$, and also with the background metric i.e., $C_{\mathfrak{g}_{1}}^{*} \cap C_{\mathfrak{g}_{2}}^{*} \cap C_{\eta}^{*} \neq \emptyset$. Furthermore, (i) $C_{\mathfrak{g}_{1}}^{*} \subseteq C_{\mathfrak{g}_{2}}^{*}$ (ii) $C_{\mathfrak{g}_{2}} \subseteq C_{\mathfrak{g}_{1}}$.

Proof.-First, we want to see whether there is a nonempty intersection among the effective cocones. The idea is to choose the parameter $\varepsilon$ "sufficiently small" so as to construct an explicit timelike covector with respect to both reciprocal effective metrics

Lemma 2: If $\varepsilon^{4}<\min _{i=1,2}\left(\left|b_{i}\right|^{-1}\right)$, there exists a covector which is timelike with respect to both reciprocal effective metrics.

Proof.-Let us consider, in the present frame, four linearly independent covectors: $\tau_{a}=(1,0,0,0)$; $x_{a}=(0,1,0,0) ; y_{a}=(0,0,1,0) ; z_{a}=(0,0,0,1)$. One obtains

$$
\begin{aligned}
\mathfrak{g}_{i}^{a b} \tau_{a} \tau_{b} & =\left(1-\varepsilon^{4} b_{i}\right)>0 \\
\mathfrak{g}_{i}^{a b} x_{a} x_{b} & =-1 \mathfrak{g}_{i}^{a b} y_{a} y_{b}=-1 \\
\mathfrak{g}_{i}^{a b} z_{a} z_{b} & =-\left(1+\varepsilon^{4} b_{i}\right)<0 .
\end{aligned}
$$

Therefore, there exists a common timelike covector $\tau_{a}$, and thus $C_{\mathfrak{g}_{1}}^{*} \cap C_{\mathfrak{g}_{2}}^{*} \cap C_{\eta}^{*} \neq \emptyset$.

To prove (i), we proceed along the same lines as in the nondegenerate case. First, from the definitions of $b_{1}$ and $b_{2}$, there follows $b_{2} \leq b_{1}$. We then consider a continuous transformation from a timelike to a spacelike covector, parametrized by $x \in[0,1]$ in the form

$$
n_{a}(x)=(1-x) \tau_{a}+x z_{a}
$$

with $\tau_{a}$ and $z_{a}$ as defined above. Again, we look for $x_{1}$ and $x_{2}$ within the range $[0,1]$, for which the covector becomes null with respect to both metrics, i.e, $g_{i}^{a b} n_{a}\left(x_{i}\right) n_{b}\left(x_{i}\right)=0$ (for $i=1,2$ ). Then,

$$
x_{i}=\frac{1-\varepsilon^{4} b_{i}}{2},
$$

which implies $x_{1} \leq x_{2}$. Consequently, $C_{\mathfrak{g}_{1}}^{*} \subseteq C_{\mathfrak{g}_{2}}^{*}{ }^{9}$ We remark that (ii) can be easily obtained along the same lines.

\section{HYPERBOLIZATIONS}

To analyze the evolutionary properties of the system (3) more closely, we shall recast it in the geometrical framework suggested by Geroch [26],

$$
K_{A}{ }^{m}{ }_{\alpha}(x, \Phi) \partial_{m} \Phi^{\alpha}+J_{A}(x, \Phi)=0,
$$

where $K_{A}{ }^{m} \alpha(x, \Phi)$ is called the principal part and $J_{A}(x, \Phi)$ stands for semilinear contributions (the explicit form of which is unnecessary for our discussion here). In this expression, capital Latin indices, $A$, stand for the space of tensorial equations; lower Latin indices, $m$, stand for spacetime indices; and Greek indices, $\alpha$, stand for for multitensorial unknowns.

Remark 3: Typically, smooth solutions of (28) are interpreted as cross sections $\Phi^{\alpha}(x)$ over a smooth fiber bundle $\mathcal{B}$, with points $\kappa=\left(x^{a}, \Phi^{\alpha}\right)$, and we interpret the fiber over $x^{a}$ as the space of allowed physical states at $x^{a}$, i.e., as the space of possible field values at that point.

Definition 1: By a hyperbolization of (28) over a submanifold $\boldsymbol{S} \in \boldsymbol{M}$, we mean a smooth symmetrizer $h^{A}{ }_{\alpha}$ such that:

(1) the field $h_{\alpha}^{A} K_{A}{ }_{\beta}{ }_{\beta}$ is symmetric in $\alpha, \beta$ in $S$

(2) there exists a covector $n_{m} \in T^{*} \boldsymbol{S}$ such that $h_{\alpha}^{A} K_{A}{ }_{\beta} n_{m}$ is positive definite.

\footnotetext{
${ }^{9}$ Notice that, in order to conclude this, it is enough to start from the common timelike covector and "move" in any direction, since we already know there is one (and just one) common null direction among the effective metrics. Thus, we argue it is not possible to find different results in different directions. As observed in the beginning of this section, once they have a nonempty intersection, one of the cones must be included on the other.
} 
If a system of first-order PDEs admits a symmetrizer satisfying the above conditions, we say that it is symmetric hyperbolic.

Once a hyperbolizer exists, standard theorems apply, and we know that, given any smooth data in a hypesurface such that $n_{a}$ is normal to it, a local solution for it would exist. Notice that, since the set of covectors $n_{a}$ for which the hyperbolizer is positive is open, we can always choose them in a neighborhood of a point so that they are surface forming. We introduce also, for later convenience, the notion of physical propagation in this context. We denote by $C_{H}^{*}$ the collection of all covectors $n_{a}$ satisfying condition (2) above. Then, $C_{H}^{*}$ is a nonempty open convex cone.

Definition 2: The "signal-propagation directions" will be given by all tangent vectors $p^{a}$, such that $p^{a} n_{a}>0$, $\forall n_{a} \in C_{H}^{*}$. The set of $p^{a}$ also forms a (nonempty) closed convex cone denoted by $C_{H}$, the "dual cone" of $C_{H}^{*}$.

Remark 4: These cones could depend, in principle, on the hyperbolization selected. But it turns out that, for most physical examples, these cones are essentially independent of hyperbolization.

For NLED, we declare $\Phi^{\alpha} \rightarrow F^{b c}$, and a closer inspection of (3) allows us to read off the principal symbol

$$
K_{A}{ }^{m}{ }_{\alpha} \rightarrow\left(-\left[g_{a}{ }^{m} b c+F_{a}{ }^{m}\left(\xi_{1} F_{b c}+\xi_{2} F_{b c}^{*}\right)+F_{a}^{*}{ }^{m}\left(\xi_{2} F_{b c}+\xi_{3} F_{b c}^{*}\right)\right], \frac{1}{2} \eta_{a}{ }^{m} b c\right),
$$

with $g_{a b c d}:=\frac{1}{2}\left(g_{a c} g_{b d}-g_{a d} g_{b c}\right)$. In what follows, we shall see that symmetric hyperbolicity holds for NLED under some basic assumptions. Basically, the latter are conditions on the Lagrangian, its derivatives, and field strengths.

\section{A. Symmetrizer}

Our first task is to find a symmetrizer for (29). In other words, we look for an $h^{A}{ }_{\alpha}$ such that $\delta \Phi^{\alpha}\left(h^{A}{ }_{\alpha} K_{A}{ }^{m}{ }_{\beta}\right) \delta \hat{\Phi}^{\beta}$ is symmetric in $\delta \Phi$ and $\delta \hat{\Phi}$. Making the identifications $\delta \Phi^{\alpha} \rightarrow X^{a b}$ and $\delta \hat{\Phi}^{\alpha} \rightarrow Y^{a b}$, with $X^{a b}$ and $Y^{a b}$ arbitrary antisymmetric tensors, one has

$$
K_{A}{ }_{\beta} \delta \hat{\Phi}^{\beta}=\left(-\left[Y_{a}{ }^{m}+A_{\mathbf{Y}} F_{a}{ }^{m}+B_{\mathbf{Y}} F_{a}{ }^{m}\right], Y_{a}{ }^{m}\right),
$$

where

$$
A_{\mathbf{Y}}:=\left[\xi_{1}(\mathbf{F} . \mathbf{Y})+\xi_{2}(\stackrel{*}{\mathbf{F}} \mathbf{Y})\right] \quad B_{\mathbf{Y}}:=\left[\xi_{2}(\mathbf{F} . \mathbf{Y})+\xi_{3}(\mathbf{F} . \mathbf{Y})\right]
$$

and $\mathbf{X} . \mathbf{Y} \equiv X^{a b} Y_{a b}$. Note that the above relations depend on the background field, the background metric, and the particular Lagrangian theory. It is natural to expect that $h_{\alpha}^{A}$ depends also on these quantities. Also, when the theory is linear, i.e., $A_{\mathbf{Y}}=B_{\mathbf{Y}}=0$, we know that

$$
h^{A}{ }_{\alpha} \delta \Phi^{\alpha}=\left(X_{q}^{a},-X_{q}^{*}\right) t^{q},
$$

where $t^{q}$ is an auxiliary smooth vector field. Therefore, the full nonlinear symmetrizer must i) reduce to (32) in the linear case and ii) depend on at least one smooth vector field $t^{q}(x)$. We claim that it is given by the formula

$$
h_{\alpha}^{A} \delta \Phi^{\alpha}=\left(X_{q}^{a},-X_{q}^{*}{ }_{q}-A_{\mathbf{X}} F^{*}{ }_{q}+B_{\mathbf{X}} F^{a}{ }_{q}\right) t^{q} .
$$

To show that it is indeed a symmetrizer, we first multiply (33) by (30). It follows that

$$
\delta \Phi^{\alpha}\left(h_{\alpha}^{A} K_{A}{ }_{\beta}{ }_{\beta}\right) \delta \hat{\Phi}^{\beta}=\left(-X_{q}^{a} Y_{a}{ }^{m}-A_{\mathbf{Y}} X^{a}{ }_{q} F_{a}{ }^{m}-B_{\mathbf{Y}} X^{a}{ }_{q} F_{a}^{*}{ }^{m}-X^{a}{ }_{q}^{*} Y_{a}^{*}{ }^{*}-A_{\mathbf{X}} F^{*}{ }_{q} Y_{a}^{*}{ }^{*}+B_{\mathbf{X}} F^{a}{ }_{q} Y_{a}^{*}{ }^{m}\right) t^{q} .
$$

Recalling that any pair of antisymmetric tensors satisfies

$$
X^{a q} Y_{a m}^{*}=-\frac{1}{2}(\mathbf{X} . \mathbf{Y}) \delta_{m}^{q}+X_{a m} Y^{a q}, \quad X^{a q} Y_{a m}^{*}=\frac{1}{2}(\stackrel{*}{\mathbf{X}} . \mathbf{Y}) \delta_{m}^{q}-X_{a m}^{*} Y^{a q},
$$

we obtain

$$
\delta \Phi^{\alpha}\left(h_{\alpha}^{A} K_{A}{ }_{\beta}\right) \delta \hat{\Phi}^{\beta}=\left(M_{q}{ }^{m}+N_{q}{ }^{m}+L_{q}{ }^{m}\right) t^{q},
$$

with 


$$
\begin{aligned}
& M_{q}{ }^{m}=+\left(X_{q}{ }^{a} Y_{q}{ }^{m}+Y_{q}{ }^{a} X_{a}{ }^{m}\right)+\frac{1}{2}(\mathbf{X} . \mathbf{Y}) \delta_{q}{ }^{m} \\
& N_{q}{ }^{m}=-\left(A_{\mathbf{Y}} X_{q}{ }^{a}+A_{\mathbf{X}} Y_{q}{ }^{a}\right) F_{a}{ }^{m}-\left(B_{\mathbf{Y}} X^{a}{ }_{q}+B_{\mathbf{X}} Y^{a}{ }_{q}\right) F_{a}{ }^{m}{ }^{m}, \\
& L_{q}{ }^{m}=+\left\{\xi_{1}(\mathbf{F} . \mathbf{X})(\mathbf{F} . \mathbf{Y})+\xi_{2}[(\mathbf{F} . \mathbf{X})(\mathbf{F} . \mathbf{Y})+(\mathbf{F} . \mathbf{Y})(\mathbf{F} . \mathbf{X})]+\xi_{3}(\mathbf{F} . \mathbf{X})(\mathbf{F} . \mathbf{Y})\right\} \delta_{q}{ }^{m} / 2,
\end{aligned}
$$

which are symmetric quantities in $\mathrm{X}$ and $\mathrm{Y}$. Note that this result is general and does not depend on the specific form of the Lagrangian. Note also that the symmetrizer splits into a main term, which coincides with Maxwell's symmetrizer and two nonlinear terms involving the background 2-form and its dual.

\section{B. Positive definiteness}

We now investigate when the symmetrizer constitutes a hyperbolization of the equations of motion. For the sake of conciseness, let us define the symmetric object

$$
H_{\alpha \beta}(t, n) \equiv h_{\alpha}^{A}(t) K_{A}{ }^{m} n_{m} .
$$

We emphasize here the linear dependence of this object with respect to both the vector $t^{a}$ (in the symmetrizer) and the covector $n_{m}$, a fact that will be important later on. We shall see that the admissible range for the quantities $\left(t^{q}, n_{m}\right)$ has a nice geometrical interpretation in terms of the effective metrics.

According to our previous definition, the system will be symmetric hyperbolic if $H_{\alpha \beta}(t, n)$ constitutes a positive definite bilinear form, i.e.,

$$
\Phi^{\alpha} H_{\alpha \beta}(t, n) \Phi^{\beta}>0,
$$

for any nonzero $\Phi^{\alpha}$. Here, $\Phi^{\alpha}$ represents an arbitrary 2-form and as such can be thought of as a vector in $\mathbb{R}^{6}$. Therefore, the family of symmetric maps $H_{\alpha \beta}(t, n): \mathbb{R}^{6} \rightarrow \mathbb{R}$. One can build a natural basis for this space by taking the six possible antisymmetrized pairs of basis elements in $T_{p}^{*} \mathbf{M}$.

From (35), we get

$$
H_{\alpha \beta}(t, n)=\left.H_{\alpha \beta}(t, n)\right|_{M}+\left.H_{\alpha \beta}(t, n)\right|_{N}+\left.H_{\alpha \beta}(t, n)\right|_{L} .
$$

A tedious but straightforward calculation yields

$$
\begin{aligned}
\left.H_{\alpha \beta}(t, n)\right|_{M}= & \left(g_{a b q[c} \delta_{d}^{m}+g_{c d q[a} \delta_{b]}^{m}+g_{a b c d} \delta_{q]}^{m}\right) t^{q} n_{m}, \\
\left.H_{\alpha \beta}(t, n)\right|_{N}= & \left(\left(\xi_{1} F_{a b}+\xi_{2} F_{a b}^{*}\right) g_{q[c} F_{d]}^{m}+\left(\xi_{1} F_{c d}+\xi_{2} F_{c d}^{*}\right) g_{q[a} F_{b]} m\right. \\
& \left.+\left(\xi_{2} F_{a b}+\xi_{3} F_{a b}^{*}\right) g_{q[c} F_{d]}^{*}+\left(\xi_{2} F_{c d}+\xi_{3} F_{c d}^{*}\right) g_{q[a} F_{b]}^{*}\right) t^{q} n_{m}, \\
\left.H_{\alpha \beta}(t, n)\right|_{L}= & \frac{1}{2}\left(\xi_{1} F_{a b} F_{c d}+\xi_{2}\left(F_{a b}^{*} F_{c d}+F_{a b}^{*} F_{c d}\right)+\xi_{3} F_{a b}^{*} F_{c d}^{*}\right)(t . n) .
\end{aligned}
$$

We now address the following. What are the conditions on the particular NLED theory (and fields) in order to have at least a pair $\left(t^{a}, n_{a}\right)$ satisfying the positivity requirement (37)? Among the symmetric hyperbolic cases, what are the sets of admissible $t^{a}$ giving rise to a hyperbolization, and what are the associated propagation cones? The answers to these questions are provided by the following theorems.

Theorem 1: The system is symmetric hyperbolic if and only if the effective cones (cocones) have a nonempty intersection, i.e., $C_{\mathfrak{g}_{1}} \cap C_{\mathfrak{g}_{2}} \neq \emptyset\left(C_{\mathfrak{g}_{1}}^{*} \cap C_{\mathfrak{g}_{2}}^{*} \neq \emptyset\right)$. This will be the case whenever

$$
\alpha_{1} \beta_{2}=1+\frac{1}{2}\left(\left(F N_{1}+G N_{2}\right)-\sqrt{N_{1}^{2}+N_{2}^{2}} \sqrt{F^{2}+G^{2}}\right)
$$$$
>0 \text {. }
$$

The strategy behind our proof is as follows: write $H_{\alpha \beta}(t, n)$ in a preferred representation, and build a mixed matrix $T_{\beta}^{\alpha}$ (by means of an auxiliary Riemannian inner product $p_{\alpha \beta}$ of $\mathbb{R}^{6}$ ), such that

$$
H_{\alpha \beta}=p_{\alpha \gamma} T_{\beta}^{\gamma}
$$

In particular, we choose $p_{\alpha \beta}=\delta_{\alpha \beta}$, with $\delta_{\alpha \beta}$ the six-dimensional Kronecker delta. We then look for the positiveness of all the eigenvalues of $T^{\alpha}{ }_{\gamma}$ which is essentially equivalent to the positive definiteness of $H_{\alpha \beta} \cdot{ }^{10}$ It turns out that the above

\footnotetext{
${ }^{10}$ The reason to introduce $p_{\alpha \beta}$ is simply because it only makes sense to talk about eigenvalues when the operator acts from one vector space to itself. It might sound like a rather technical subtlety, but is not, since there is not a unique (or natural) metric to raise the index here. There is an arbitrariness involved on the inner product, and the resulting eigenvalues are not going to be covariantly definded in general; one is implicitly introducing a coordinate dependence on the election of a particular $p_{\alpha \beta}$. Nevertheless, the positive character of the eigenvalues is in fact invariant, and it will guarantee the positive definiteness of $H_{\alpha \beta}$, as long as $p_{\alpha \beta}$ is an inner product.
} 
requirement imposes a restriction on the allowed values of the background invariants (and on the theories, through $\xi_{i}$ ); such restriction is precisely (39), and it is equivalent to the statement that the effective cones do intersect each other. This result reveals an interesting geometrical aspect of NLED regarding hyperbolicity. Also, it provides a simple diagnostic tool for computing whether such a geometrical property holds in a particular situation. Note that (39) is a local condition which contains information on the particular theory but also depends on the background solution and space-time point one is looking at. Thus, it might be possible to have theories which are symmetric hyperbolic only for a reduced subset of field configurations.

Theorem 2: A theory satisfying (39) admits a collection of symmetrizers parametrized by vectors $t^{a}$ such that $t^{a} \in C_{\mathfrak{g}_{1}} \cap C_{\mathfrak{g}_{2}}=C_{\mathfrak{g}_{2}}$. Regardless of the particular choice, the resulting propagation cone $C_{H}$ is given by the closure of the union of the effective cones, i.e, $C_{H}=\bar{C}_{\mathfrak{g}_{1}} \cup \bar{C}_{\mathfrak{g}_{2}}=\bar{C}_{\mathfrak{g}_{1}}$.

The starting point underlying the second proof is the construction of an explicit pair $\left(t_{o}^{a}, n_{a}^{o}\right)$ satisfying the positivity condition. We then show this particular couple necessarily lies in the intersections of the cones (cocones) engendered by the effective metrics. Then, by looking at the determinant of $T^{\alpha}{ }_{\gamma}$, we will argue how much we can extend the vector $t_{o}^{a}$ and the covector $n_{a}^{o}$ without losing the positive character of the eigenvalues. It turns out that the limits for extending them are just the closures of the above-mentioned intersections. Finally, once one finds all possible covectors $n_{a}$, a simple computation yields the propagation cone $C_{H}$, which we find to be $C_{H}=\bar{C}_{\mathfrak{g}_{1}} \cup \bar{C}_{\mathfrak{g}_{2}}$, and, as a consequence of proposition 2 , there follows $C_{H}=\bar{C}_{\mathfrak{g}_{1}}$.

\section{PROOF OF THE MAIN THEOREMS}

To begin with, note that $H_{\alpha \beta}(t, n)$ is linear with respect to $t^{a}$ and $n_{a}$. Thus, once a pair $\left(t_{o}^{a}, n_{a}^{o}\right)$ satisfying $H_{\alpha \beta}\left(t_{o}, n^{o}\right)>0$ is found, then (by keeping one of them fixed, say $t_{o}^{a}$ ) there will exist a neighborhood (around the other, $n_{a}^{o}$ ) for which $H_{\alpha \beta}\left(t^{o}, n\right)$ will remain positive definite for all $n$ in that neighborhood. These neighborhoods necessarily define open and convex sets which characterize them as cones according to our definition.

\section{A. Nondegenerate $\boldsymbol{F}_{a b}$}

We start by writing $H_{\alpha \beta}(t, n)$ in a convenient representation. Our choice is irrelevant from the conceptual perspective but important from the operational point of view. We rely on the same frame used in Sec. II. C and consider as a basis for $\mathbb{R}^{6}$ all antisymmetrized pairs of the covectors $\tau_{a}, x_{a}, y_{a}, z_{a}$ (see Appendix A). A well-established algebraic result [56] states that the positive definiteness of $H_{\alpha \beta}(t, n)$ is equivalent to the positivity of all eigenvalues of a matrix $T_{\beta}^{\alpha}$, related via (40), for any Riemannian inner product $p_{\alpha \beta}$.
Thus, we seek for a specific pair $\left(t_{o}^{a}, n_{a}^{o}\right)$, rendering all eigenvalues positive. Unfortunately, we were not able to explicitly calculate them for the most general pair. However, assuming that $t_{o}^{a}$ and $n_{a}^{o}$ lie within the plane defined by the principal null directions, calculations simplify considerably. In the chosen frame, this hypothesis is equivalent to

$$
t_{o}^{a}=\left(t_{0}, 0,0, t_{3}\right) \quad ; \quad n_{a}^{o}=\left(n_{0}, 0,0, n_{3}\right) .
$$

For such pairs, the six eigenvalues $\lambda_{i}$ were computed using Mathematica and are given by

$$
\begin{aligned}
\lambda_{1,2} & =\left(n_{0}-n_{3}\right)\left(t_{0}+t_{3}\right) \\
\lambda_{3,4} & =\left(n_{0}+n_{3}\right)\left(t_{0}-t_{3}\right) \\
\lambda_{5} & =\left(n_{0} t_{0}+n_{3} t_{3}\right) \alpha_{1} \alpha_{2} \\
\lambda_{6} & =\left(n_{0} t_{0}+n_{3} t_{3}\right) \beta_{1} \beta_{2} .
\end{aligned}
$$

Consequently, $\lambda_{5}$ and $\lambda_{6}$ will be positive if $\operatorname{sign}\left(\alpha_{1} \alpha_{2}\right)=\operatorname{sign}\left(\beta_{1} \beta_{2}\right)$. Equivalently, we have

$$
0<\alpha_{1} \alpha_{2} \beta_{1} \beta_{2}=\Omega_{1} \Omega_{2} \text {. }
$$

These relations imply the following: i) $\operatorname{sign}\left(\Omega_{1}\right)=$ $\operatorname{sign}\left(\Omega_{2}\right)$, which, as we have seen, means that the two cones have a nonempty intersection, and ii) recalling that $\alpha_{1} \beta_{2} \leq 1 \leq \alpha_{2} \beta_{1}$ (from Lemma 1 in Sec. II. C), one has $\alpha_{1} \beta_{2}>0$, which is precisely expression (39) from Theorem 1. Under these assumptions, there are only two possible cases to analyze: $\Omega_{i}>0$, which corresponds to Fig. 1a (Sec. II. C), and $\Omega_{i}<0$, as illustrated in Fig. 1c. These two cases are considered separately below:

(1) If $\Omega_{i}>0$, we choose $t_{o}^{a}=(1,0,0,0)$ and $n_{a}^{o}=(1,0,0,0)$. And we get

$$
\lambda_{1,2,3,4}=1 \quad ; \quad \lambda_{5}=\alpha_{1} \alpha_{2}>0 \quad ; \quad \lambda_{6}=\beta_{1} \beta_{2}>0 .
$$

(2) If $\Omega_{i}<0$, we choose $t_{o}^{a}=(0,0,0,1)$ and $n_{a}^{o}=(0,0,0,1)$. Obtaining [once the symmetrizer is multiplied by $(-1)]$

$$
\lambda_{1,2,3,4}=1 \quad ; \quad \lambda_{5}=-\alpha_{1} \alpha_{2}>0 ; \lambda_{6}=-\beta_{1} \beta_{2}>0 .
$$

Thus, we conclude that (39) is a sufficient condition for the system to be symmetric hyperbolic. To prove it is also necessary, we still need to justify the restriction of $\left(t_{o}^{a}, n_{a}^{o}\right)$ to those lying on the PNDs plane. We will return briefly to complete this part of the proof. For the time being, we now concentrate on the question regarding how far the neighborhoods of $\left(t_{o}^{a}\right.$, and $\left.n_{a}^{o}\right)$ can be extended, while still preserving the positivity condition. We already discard the spacelike part (with respect to the effective metrics) of the null plane, for in this case, some eigenvalues become negative. 
The determinant of $T_{\beta}^{\alpha}$ was calculated using Mathematica and is given by

$$
\begin{aligned}
\operatorname{det}\left(T^{\alpha}{ }_{\beta}\right)= & \Omega_{1} \Omega_{2}\left(\mathfrak{g}_{1}^{a b} n_{a} n_{b}\right)\left(\mathfrak{g}_{2}^{a b} n_{a} n_{b}\right)\left(n_{a} t^{a}\right)^{2} \\
& \times\left(\mathfrak{g}_{a b}^{1} t^{a} t^{b}\right)\left(\mathfrak{g}_{a b}^{2} t^{a} t^{b}\right) \\
= & \lambda_{1} \lambda_{2} \ldots \lambda_{6}
\end{aligned}
$$

for the generic vector $t^{a}$ and covector $n_{a}$. This result is at the base of the following discussions.

First, notice that the vector $t_{o}^{a}$ (and covector $n_{a}^{o}$ ) we find above belongs to the intersection of the two cones (cocones). In other words, $t_{o}^{a} \in C_{\mathfrak{g}_{1}} \cap C_{\mathfrak{g}_{2}}$ and $n_{a}^{o} \in C_{\mathfrak{g}_{1}}^{*} \cap C_{\mathfrak{g}_{2}}^{*} \cdot{ }^{11}$ Now, whenever an eigenvalue becomes zero, one of the following must hold: (a) $n_{a}$ is a null covector of either $\mathfrak{g}_{1}^{a b}$ or $\mathfrak{g}_{2}^{a b}$, (b) $t^{a}$ is a null vector of either $\mathfrak{g}_{a b}^{1}$ or $\mathfrak{g}_{a b}^{2}$, or (c) $t^{a} n_{a}=0$.

Proposition 4: If $t^{a} \in C_{\mathfrak{g}_{1}} \cap C_{\mathfrak{g}_{2}}$ and $n_{a} \in C_{\mathfrak{g}_{1}}^{*} \cap C_{\mathfrak{g}_{2}}^{*}$, then $t^{a} n_{a}>0$.

Proof.-We rely on previous results from Sec. II. C, in particular, Proposition 2, which states (i) $C_{\mathfrak{g}_{1}}^{*} \subseteq C_{\mathfrak{g}_{2}}^{*}$ and (ii) $C_{\mathfrak{g}_{2}} \subseteq C_{\mathfrak{g}_{1}}$. Thus,

$$
\begin{aligned}
& C_{\mathfrak{g}_{1}} \cap C_{\mathfrak{g}_{2}} \equiv C_{\mathfrak{g}_{2}} \\
& C_{\mathfrak{g}_{1}}^{*} \cap C_{\mathfrak{g}_{2}}^{*} \equiv C_{\mathfrak{g}_{1}}^{*} \subseteq C_{\mathfrak{g}_{2}}^{*} .
\end{aligned}
$$

It is straightforward to see now that any covector $n_{a} \in$ $C_{\mathfrak{g}_{1}}^{*} \subseteq C_{\mathbf{g}_{2}}^{*}$ will satisfy (recalling the definition of dual cone) that $t^{a} n_{a}>0$, for any vector $t^{a} \in C_{\mathfrak{g}_{2}}$.

From this result, we conclude that the set of all the $t^{a}$ for which the symmetrizer is positive is just $C_{\mathfrak{g}_{1}} \cap C_{\mathfrak{g}_{2}} \equiv C_{\mathfrak{g}_{2}}$ and similarly that the set of all $n_{a}$ is given by $C_{\mathfrak{g}_{1}}^{*} \cap C_{\mathfrak{g}_{2}}^{*} \equiv C_{\mathfrak{g}_{1}}^{*}$. As a corollary, and according to Geroch's definition of a propagation cone (we gave in Sec. III, Definition 2), we find

$$
C_{H}=\bar{C}_{\mathfrak{g}_{1}} \equiv \bar{C}_{\mathfrak{g}_{1}} \cup \bar{C}_{\mathfrak{g}_{2}}
$$

This concludes the proof of Theorem 2 for a nondegenerate $F_{a b}$. To complete the demonstration of Theorem 1, we proceed by contradiction.

To show (39) is also a necessary condition for (symmetric) hyperbolicity, we assume $C_{\mathfrak{g}_{1}} \cap C_{\mathfrak{g}_{2}}=\emptyset$ and that there exists a pair $\left(t_{o}^{a}, n_{a}^{o}\right)$ satisfying $\lambda_{i}>0, \forall i=1,2, \ldots, 6$. This will lead us to a contradiction. Indeed, if $t_{o}^{a}$ (or $n_{a}^{o}$ ) lies outside both cones (cocones), then the resulting set of allowed vectors (covectors) acoording to (41) does not configure a convex cone. In fact, Eq. (41) directly implies that if such a vector (covector) existed one would be able to

\footnotetext{
${ }^{11}$ In our frames, this can be seen in a rather direct way for each of the two situations, namely, $\Omega_{i}>0$ and $\Omega_{i}<0$. That is why we believe it is not necessary to further justify this statement.
}

keep the positivity of the symmetrizer by reflecting the corresponding vector through the preferred plane. However, it turns out that their sum would fall into a part of the null plane in which the system is not hyperbolic. Thus, the set of vectors would not characterize a cone according to our definition, which is a contradiction. Therefore, both $t_{o}^{a}$ and $n_{a}^{o}$ must belong to one of their respective cones/cocones. The contradiction came from assuming one could find a pair $\left(t_{o}^{a}, n_{a}^{o}\right)$ satisfying $\lambda_{i}>0, \quad \forall i=1,2, . ., 6$ for the cases in which the cones do not intersect. Thus, Eq. (39) is also a necessary condition which finishes the proof of THM 1 (for the nondegenerate cases).

\section{B. Degenerate $\boldsymbol{F}_{\boldsymbol{a} b}$}

To complete our proofs, we consider here the cases where the background field is degenerate. It turns out that these cases are much simpler, since (as we have already seen) there is always a nonempty intersection of the two cones. Thus, it only remains to be proven that there exists a common timelike vector $t_{o}^{a}$ and covector $n_{a}^{o}$ (respect to both effective metrics) for which the six eigenvalues $\lambda_{i}$ of $T^{\alpha}{ }_{\beta}$ are positive. We can do this explicitly, using the results from Sec. II. C. Recall that a common timelike vector and covector were found, which in our particular frame reads

$$
t_{o}^{a}=(1,0,0,0) ; \quad n_{a}^{o}=(1,0,0,0) .
$$

Computing the eigenvalues for this pair, one obtains

$$
\begin{aligned}
& \lambda_{1}=1+\varepsilon^{4}\left|b_{1}\right|>0 \\
& \lambda_{2}=1-\varepsilon^{4}\left|b_{1}\right|>0 \\
& \lambda_{3}=1+\varepsilon^{4}\left|b_{2}\right|>0 \\
& \lambda_{4}=1-\varepsilon^{4}\left|b_{2}\right|>0 \\
& \lambda_{5}=\lambda_{6}=1 .
\end{aligned}
$$

Then, for the second part of the proof, we follow the same lines as for the nondegenerate case. Notice that expressions (41) and Proposition 4 also apply in the degenerate case. In addition, we have provided (in Sec. II C) the analog of Proposition 2, namely, Proposition 3. Therefore, everything follows identically as before and as such concludes the proofs of Theorems 1 and 2 .

\section{CONSTRAINTS}

The symmetrizer $h^{A}{ }_{\alpha}$ may be understood as map from the space of equations (indexed by " $A$ ") to the space of unknowns (indexed by " $\alpha$ "). In other words, it selects from the entire set of first-order equations some combinations of equations which we can evolve along some direction which we usually relate to time. What are the remaining equations the symmetrizer does not capture? For the system to be 
consistent, they should not be of the evolution type, for in that case, they are either a linear combination of the ones already selected by the symmetrizer or they would be incompatible with the previously chosen evolution. In other words, they must be satisfied automatically once they are satisfied initially; i.e., they should be what we normally call the constraints. In Geroch's formalism, a constraint is a tensor $c^{A n}$ such that

$$
c^{A(n} K_{A}{ }^{m)}{ }_{\alpha}=0 .
$$

When this formalism is applied to the equations of nonlinear electrodynamics, we obtain a linear space of constraints characterized by vectors $(x, y)$ in $\mathbb{R}^{2}$ of the form $c^{A n}=\left(x g^{a n}, y g^{a n}\right)$. To check that this $c^{A n}$ does indeed satisfy (43), we combine it with the principal symbol to obtain

$$
c^{A n} K_{A}{ }_{\alpha}{ }_{\alpha}=-x\left\{\frac{1}{2} g^{n m b c}+F^{n m}\left(\xi_{1} F^{b c}+\xi_{2} F^{b c}\right)+\stackrel{*}{F^{n m}}\left(\xi_{2} F^{b c}+\xi_{3} F^{b c}\right)\right\}+\frac{1}{2} y \eta^{n m b c},
$$

which is antisymmetric in the quantities $n$ and $m$. The constraints are complete in the sense that the dimension of evolution equations provided by the symmetrizer plus the dimension of constraint equations gives the correct number of PDEs. Note that, contrarily to $K_{A}{ }^{m}{ }_{\alpha}$, the tensor $c^{A n}$ does not depend on the electromagnetic field $F_{a b}$ and coincides with those of the linear theory. We now show that this twodimensional system is integrable, i.e., that it satisfies

$$
\begin{aligned}
\nabla_{n}\left(c^{A n} K_{A}{ }^{m}{ }_{\alpha} \nabla_{m} \Phi^{\alpha}\right)= & \nabla_{n}\left(c^{A n} K_{A}{ }^{m}{ }_{\alpha}\right) \nabla_{m} \Phi^{\alpha} \\
& +\nabla_{n} \nabla_{m}\left(c^{A n} K_{A}{ }^{m}{ }_{\alpha} \Phi^{\alpha}\right)=0
\end{aligned}
$$

identically. The second-derivative term drops out, as a consequence of (43), and so we are left with an algebraic equation in the first derivatives, of the form

$$
\frac{\partial}{\partial \Phi^{\beta}}\left(c^{A n} K_{A}{ }_{\alpha}{ }_{\alpha}\right) \nabla_{m} \Phi^{\alpha} \nabla_{n} \Phi^{\beta}=0 .
$$

This is an integrability condition for the equations as a whole. If it holds as a trivial algebraic consequence of the equations of motion, we say that our constraint is integrable. To show that this is indeed the case for any NLED derived from a Lagrangian, it is convenient to rewrite the equations of motion in the form

$$
\nabla_{m}\left(\tilde{K}_{A}{ }^{m}{ }_{\alpha} \Phi^{\alpha}\right)=0
$$

with $\tilde{K}_{A}{ }^{m}{ }_{\alpha} \rightarrow \frac{1}{2}\left(\mathcal{L}_{F} g_{a}{ }^{m b c}+\mathcal{L}_{G} \eta_{a}{ }^{m b c}, \eta_{a}{ }^{m b c}\right)$. Multiplying by $c^{A n} \nabla_{n}$ and noting that $c^{A n}$ commutes with the derivatives, we obtain for the left-hand side

$$
\nabla_{n} \nabla_{m}\left(c^{A n} \tilde{K}_{A}{ }^{m}{ }_{\alpha} \Phi^{\alpha}\right) .
$$

Now, because $c^{A n} \tilde{K}_{A}{ }^{m}{ }_{\alpha}$ is also antisymmetric in $n$ and $m$, this quantity is identically zero. Thus, Eq. (46) holds trivially, and the constraints are therefore integrable; i.e., they remain true on the whole domain of dependence provided they were so at the initial time. When the constraints are integrable, by studying the properties of the compound hyperbolic PDE system, it follows that all the well-posed results for symmetric hyperbolic systems also apply in the presence of constraints.

\section{EXAMPLES}

In this section, we discuss several examples illustrating the power of the results discussed so far. The latter serve as simple diagnostic tools in testing whether well posedness (hyperbolicity) holds and what the associated physical propagation cones are. In particular, if $\alpha_{1} \beta_{2}>0$, Theorem (1) guarantees a nontrivial intersection between the effective cones and therefore hyperbolicity. When this condition is fulfilled, we shall check whether these cones intersect the background cone or not and whether the propagation speeds (given by $\bar{C}_{\mathfrak{g}_{1}}$ ) are subor superluminal.

We will perform this analysis based on $\gamma_{1}$ for nondegenerate cases and $b_{1}$ for degenerate ones. It follows from Propositions 1 and 2 that: (a) when $\gamma_{1}<1$, then $\bar{C}_{\eta}\left(\operatorname{or} \bar{C}_{\sigma}\right) \subseteq \bar{C}_{\mathfrak{g}_{1}} ;\left(\right.$ b) when $\gamma_{1}=1$, then $\bar{C}_{\mathfrak{g}_{1}}=\bar{C}_{\eta}\left(\operatorname{or} \bar{C}_{\sigma}\right)$ the effective metric becomes in the background (of a rotation of it); (c) finally in the sub-luminal case $1<\gamma_{1}$ then $\bar{C}_{\mathfrak{g}_{1}} \subseteq \bar{C}_{\eta}$ or $\left(\bar{C}_{\sigma}\right)$. Notice that, since all the metrics share at least one null direction along that direction, all propagation speeds coincide, so we always have some directions with speed of light propagation.

For degenerate $F_{a b}$, the causal structure depends on $b_{1}$, therefore, it follows from Propositions 3 , there are 3 possibilities: (a) $b_{1}<0$ then $\bar{C}_{\mathfrak{g}_{1}} \subseteq \bar{C}_{\eta}$; (b) $b_{1}=0$ then $\bar{C}_{\eta}=\bar{C}_{\mathfrak{g}_{1}} ;$ (c) $0<b_{1}$ then $\bar{C}_{\eta} \subseteq \bar{C}_{\mathfrak{g}_{1}}$.

In the next examples, we shall use a frame in which $\vec{E} \| \vec{H}$ (A3); in that case, $\frac{\sqrt{F^{2}+G^{2}}-F}{4}=E^{2}$ and $\frac{\sqrt{F^{2}+G^{2}}+F}{4}=H^{2}$.

\section{A. Born-Infeld}

The Born-Infeld theory is the paramount example of nonlinear electrodynamics. It was proposed to remove the divergence of the electron's self-energy at the classical level. The idea was to use a nonlinear generalization to 
Maxwell's theory, which deviates from it at very strong fields. It naturally introduces a cutoff $\beta$ limiting of maximum electric fields around a static charge, thus avoiding the singularity at $r \rightarrow 0$. The Lagrangian expression is

$$
\mathcal{L}=\beta^{2}\left(\sqrt{1+\frac{F}{2 \beta^{2}}-\frac{G^{2}}{16 \beta^{4}}}+1\right) .
$$

One interesting property of this theory is the absence of birefringence; since $N_{1}=N_{2}=0$ [47], the two effective metrics are identical, and so are the propagation speeds of all physical modes. In this case, the cones' intersection is obvious, and the systems is symmetric hyperbolic. In addition, the propagation cone is contained in the light cone of the space-time metric, so propagation speeds are lower than or equal to the speed of light:

(i) Nondegenerate case $F^{2}+G^{2} \neq 0$ :

Before starting the hyperbolicity analysis, it is important to note that, when $\beta^{2} \rightarrow \frac{\sqrt{F^{2}+G^{2}}-F}{4}$, $\frac{\partial \mathcal{L}}{\partial F} \rightarrow \infty$, and if $\beta^{2}<\frac{\sqrt{F^{2}+G^{2}}-F}{4}$, then $\mathcal{L}$ becomes complex. Thus, we shall restrict attention to the range $\frac{\sqrt{F^{2}+G^{2}}-F}{4}=E^{2}<\beta^{2}$, which is consistent with the original idea of a limited electric field strength in the Born-Infeld theory.

The effective metrics are conformally related, $g_{2}^{a b}=\left(\frac{-G^{2}+16 \beta^{4}+8 F \beta^{2}}{4\left(2 \beta^{2}+F\right)^{2}}\right) g_{1}^{a b}$, and it easy to check that $0<\alpha_{1} \beta_{2}=1$ (and $0<\alpha_{1} \alpha_{2} \beta_{1} \beta_{2}=1$ ), so, as we have said, the theory is symmetric hyperbolic.

In addition, $\Omega_{1}=\frac{1}{\Omega_{2}}=4 \frac{\left(2 \beta^{2}+F\right)^{2}}{16\left(\beta^{2}+H^{2}\right)\left(\beta^{2}-E^{2}\right)}>0$, and thus when $E^{2}<\beta^{2}$, the effective metrics have cone intersections with the background metric. We compute now

$$
\gamma_{1}^{2}=\left|\frac{\left(1+\frac{H^{2}}{\beta^{2}}\right)}{\left(1-\frac{E^{2}}{\beta^{2}}\right)}\right|
$$

and conclude that $1<\gamma_{1}^{2}$, i.e., it is a subluminal case, and only propagations speeds up that of light are allowed.

In the limit of weak fields, $E^{2}, H^{2} \ll \beta^{2}, \gamma_{1} \rightarrow 1$, and so, as expected, the theory becomes closer to Maxwell's.

(ii) Degenerate case $F^{2}+G^{2}=0$ :

As we have established, degenerate cases are always hyperbolic, and we can check the cone intersection. In this case, using (25), $g_{1}^{a b}=g_{2}^{a b}$, with $b_{1,2}=-\frac{1}{\beta^{2}}<0$, and the propagation speeds are lower than light.

\section{B. Toy model 1}

The present example, like the Born-Infeld one, has only one effective metric $\left(N_{1}=N_{2}=0\right)$, so it is symmetric hyperbolic for any value of the fields, but unlike the former, the effective metric cone never intersects the background metric cone [see Fig. 1c]. Its Lagrangian is given by

$$
\mathcal{L}=\frac{F}{G} \text {. }
$$

The theory is not defined for $G=0$ since $\xi_{2}, \xi_{3}, a, Q$, and $R$ blow up in this limit, so the degenerate case will not be discussed.

The effective metric definitions (12) we are using also go bad at $F=0$, but they can be rescaled (see footnote 2 ) so that they become finite. We can use these re-scaled metrics in place of the former ones for this case, and obtain the same results as before. We obtain $\bar{g}_{2}^{a b}=\left(-\frac{16}{G^{2}}\right) \bar{g}_{1}^{a b}$, so they are conformally related and therefore share the same cone, implying the system is symmetric hyperbolic. In addition, $\bar{\Omega}_{1}=\frac{1}{\bar{\Omega}_{2}}=-H^{2} E^{2}<0$, so the effective metrics cone has no intersection with the background metrics cone. This implies that initial data must be given in spacelike hypersurface, for the effective metrics, and evolution occurs in their temporal directions. Note however, that the hypersurfaces are timelike for the background metric, showing that this metric does not play any role in the hyperbolicity properties of the theory.

\section{Electrodynamics from Kaluza-Klein theory}

We shall analyze three examples introduced in Ref. [57]. The authors start from the Kaluza-Klein metrics in $5=d+1$ dimensions and add a Gauss-Bonnet term (that in five dimensions is not a topological invariant) to the Einstein-Hilbert action. They arrive at an effective electrodynamics theory in $d=3+1$ dimensions. The resulting Lagrangian is

$$
\mathcal{L}=-\frac{1}{4} F+\frac{1}{16} \gamma\left((b-1) F^{2}-\frac{3}{2} G^{2}\right)
$$

with $\gamma$ a perturbation parameter from the Lagrangian, associated with Gauss-Bonnet terms, which will be interpreted as a function of physical quantities $\left(e, m_{e}, \hbar, c\right)$, and $b$ a parameter associated to a term in the action quadratic in Ricci scalar. This last parameter is chosen in order to avoid ghost propagation and will give rise to very different electromagnetic theories.

The symmetric hyperbolic condition is

$$
\begin{aligned}
& \alpha_{1} \alpha_{2} \beta_{1} \beta_{2} \\
& \quad=\frac{\left((F(1-4 b) \gamma+4)^{2}-(5 \gamma-2 b \gamma)^{2}\left(F^{2}+G^{2}\right)\right)}{(2 F \gamma-2 F b \gamma+4) .^{2}}>0 .
\end{aligned}
$$

We shall check under which conditions this is fulfilled in the following examples. 


\section{Gauss-Bonnet electrodynamics}

For this theory, $b=1$, and it is interpreted as the firstorder string theory corrections to general relativity [58].

The symmetric hyperbolic condition becomes

$$
\alpha_{1} \alpha_{2} \beta_{1} \beta_{2}=\left(1-3 H^{2} \gamma\right)\left(1+3 \gamma E^{2}\right)>0 .
$$

Because $\operatorname{sign}(\gamma)$ is not defined, and this gives rise to different hyperbolicity conditions and different effective metrics, we need to study each case in particular:

(1) If $\gamma>0$, condition (50) implies $H^{2}<\frac{1}{3 \gamma}$.

(i) Nondegenerate case.-The effective metrics have cone intersections with background metric ones because

$$
\Omega_{1}=\left(1-3 H^{2} \gamma\right)\left(1+3 \gamma E^{2}\right)>0 .
$$

In addition, propagation faster than light is allowed, and this is so even for the degenerate case, as we will check,

$$
1>\gamma_{1}=\frac{1-3 H^{2} \gamma}{1+3 \gamma E^{2}}>0
$$

(ii) Degenerate case.

$$
b_{1}=3 \gamma>0 \text {. }
$$

(2) If $\gamma<0$, the dominant energy condition is satisfied, and condition (50) implies $E^{2}<\frac{1}{3|\gamma|}$ in concordance with Gibbons and Herdeiro.

The effective metric $\mathfrak{g}_{1}$ is conformal to the background metric, so they have the same cone:

(i) Nondegenerate case.

$$
g_{1}^{a b}=\left(1-3 \gamma H^{2}+3 \gamma E^{2}\right) \eta^{a b} .
$$

(ii) Degenerate case.

$$
g_{1}^{a b}=\eta^{a b} .
$$

\section{Born-Infeld to second order}

For $b=-\frac{1}{2}$ and $\gamma \propto \beta^{2}$, Eq. (49) approximates the BornInfeld Lagrangian to second order, so we recover birefringence:

(i) Nondegenerate case.-The condition for hyperbolicity is

$$
\alpha_{1} \alpha_{2} \beta_{1} \beta_{2}=\frac{16\left(1+3 \gamma H^{2}\right)\left(1-3 \gamma E^{2}\right)}{\left(\left(1+3 \gamma H^{2}\right)+\left(1-3 \gamma E^{2}\right)\right)^{2}}-3>0 .
$$

Then, one can prove the last expression is positive when

$$
\frac{1}{3}<\frac{\left(1+3 \gamma H^{2}\right)}{\left(1-3 \gamma E^{2}\right)}<3
$$

This implies the nonempty cones intersection of the background with effective metrics

$$
\Omega_{1}=\frac{\left(3 \gamma H^{2}+3 \gamma E^{2}+2\right)^{2}}{\left(3 \gamma H^{2}-3 \gamma E^{2}+2\right)^{2}} \frac{\left(3-\frac{\left(1+3 \gamma H^{2}\right)}{\left(1-3 \gamma E^{2}\right)}\right)}{\left(1+\frac{\left(1+3 \gamma H^{2}\right)}{\left(1-3 \gamma E^{2}\right)}\right)}>0 .
$$

Moreover,

$$
\gamma_{1}=\frac{3-\frac{\left(1+3 \gamma H^{2}\right)}{\left(1-3 \gamma E^{2}\right)}}{1+\frac{\left(1+3 \gamma H^{2}\right)}{\left(1-3 \gamma E^{2}\right)}}
$$

In the symmetric hyperbolic range:

If $0<\gamma \Rightarrow 1<\frac{\left(1+3 \gamma H^{2}\right)}{\left(1-3 \gamma E^{2}\right)}<3 \Rightarrow 0<\gamma_{1}<1$ the theory allows superluminal propagations.

If $\gamma=0 \Rightarrow$ the system reduces to Maxwell theory.

If $\gamma<0 \Rightarrow \frac{1}{3}<\frac{\left(1+3 \gamma H^{2}\right)}{\left(1-3 \gamma E^{2}\right)}<1 \Rightarrow 1<\gamma_{1}$ the theory allows only subluminal propagations, except in the preferred null direction where is luminal.

(ii) Degenerate case.

$$
b_{1}=3 \gamma \text {. }
$$

If $\gamma>0$, then $b_{1}>0$, and propagations faster than light are allowed.

If $\gamma=0$, then $b_{1}=0$, and we recover Maxwell's theory.

If $\gamma<0$, then $b_{1}<0$, and propagations up to the speed of light are allowed (the propagation speed along the unique null direction of $F_{a b}$ will be that of light).

\section{Euler-Heisenberg}

For $b=\frac{1}{7}$ and $\gamma \propto \alpha$, the fine structure constant, the Euler-Heisenberg theory becomes the effective Lagrangian for QED due to one-loop corrections [2]:

(i) Nondegenerate case.

$$
\begin{aligned}
\alpha_{1} \alpha_{2} \beta_{1} \beta_{2}= & \frac{270}{\left(6\left(\frac{\left(7+3 \gamma H^{2}\right)}{7-3 \gamma E^{2}}+1\right)-\frac{63}{7-3 \gamma E^{2}}\right)^{2}} \\
& \times\left(\frac{6}{5}-\frac{\left(7+3 \gamma H^{2}\right)}{\left(7-3 \gamma E^{2}\right)}\right) \\
& \times\left(\frac{\left(7+3 \gamma H^{2}\right)}{\left(7-3 \gamma E^{2}\right)}-\frac{5}{6}\right)>0 .
\end{aligned}
$$

Then, the systems is symmetric hyperbolic when

$$
\frac{5}{6}<\frac{\left(7+3 \gamma H^{2}\right)}{\left(7-3 \gamma E^{2}\right)}<\frac{6}{5} .
$$


The analysis of the propagation velocity in this case will not be studied due to its difficulty.

(ii) Degenerate case.

$$
b_{1}=\frac{33}{14} \gamma+\frac{9}{14}|\gamma|
$$

If $\gamma>0$, then $b_{1}>0$, and propagations higher than light are allowed.

If $\gamma=0$, we recovery Maxwell's theory.

If $\gamma<0$, then $b_{1}<0$, and propagations up to the speed of light are allowed.

\section{Euler-Heisenberg II}

We present another approach given in Ref. [59]; it is a strong field approximation for the Euler-Heisenberg action, with the Lagrangian

$$
L=-\frac{1}{4} \kappa F|F G|^{\frac{\delta}{2}}
$$

with $\kappa=E_{c}^{-2 \delta}$, a critical field, and $\delta=\frac{1}{3}\left(\frac{e^{2}}{4 \pi \hbar c \epsilon_{0}}\right)$ for spinors $\mathrm{QED}$ and $\delta_{S}=\frac{\delta}{4}$ for scalar QED.

The degenerate cases are not well defined because some quantities blow up.

For the nondegenerate case, we define

$$
y_{ \pm}=\frac{-\left(6 \delta^{4}+44 \delta^{3}+92 \delta^{2}+64 \delta+16\right) \pm \sqrt{16(\delta+2)^{3}(2 \delta+1)^{2}\left(6 \delta^{2}+5 \delta+2\right)}}{2\left(16 \delta+28 \delta^{2}-9 \delta^{4}\right)} .
$$

Thus, the system is symmetric hyperbolic when we have the following:

(i) If $\left(16 \delta+28 \delta^{2}-9 \delta^{4}\right)>0$,

$$
\frac{F^{2}}{G^{2}}<y_{-} \mathrm{o} y_{+}<\frac{F^{2}}{G^{2}}
$$

(ii) If $\left(16 \delta+28 \delta^{2}-9 \delta^{4}\right)<0$,

$$
y_{-}<\frac{F^{2}}{G^{2}}<y_{+}
$$

\section{E. Toy model 2}

We consider an arbitrary Lagrangian as function only of $F$,

$$
\mathcal{L}=\mathcal{L}(F)
$$

Then, $\xi_{2}=\xi_{3}=0$ and $b_{1}:=\left(\xi_{1}+\left|\xi_{1}\right|\right), b_{2}=\left(\xi_{1}-\left|\xi_{1}\right|\right)$.

(i) Nondegenerate case.-We will see that the hyperbolicity condition is

$$
-\frac{1}{2 H^{2}}<\xi_{1}<\frac{1}{2 E^{2}}
$$

(1) If $\xi_{1} \geq 0$, the systems is symmetric hyperbolic if $0<\alpha_{1} \beta_{2}=1-2 \xi_{1} E^{2}$; then,

$$
0 \leq \xi_{1}<\frac{1}{2 E^{2}}
$$

In addition,

$$
\gamma_{1}=\frac{1-2 \xi_{1} E^{2}}{1+2 \xi_{1} H^{2}}
$$

If $\xi_{1}=0$ (and $\gamma_{1}=1$ ), the theory behaves as Maxwellian, the birefringence effect disappears, and the propagation is given for the background cone. If $0<\xi_{1}<\frac{1}{2 E^{2}}$, then $\gamma_{1}<1$, and propagations faster than speed of light are allowed.

Moreover,

$$
\Omega_{1}=\left(1-2 \xi_{1} E^{2}\right)\left(1+2 \xi_{1} H^{2}\right)>0 ;
$$

then, the effective metric has a cone intersection with the background metric.

(2) If $\xi_{1}<0$, the systems is symmetric hyperbolic if $0<\alpha_{1} \beta_{2}=1+2 \xi_{1} H^{2}$,

$$
-\frac{1}{2 H^{2}}<\xi_{1}<0,
$$

and cone propagation is given for the background cone,

$$
\gamma_{1}=1 \Omega_{1}=1
$$

(i) Degenerate case.

If $\xi_{1}>0$ then $b_{1}=2 \xi_{1}>0$ and propagation faster than light are allowed

If $\xi_{1}<0$ then $b_{1}=0$ the background metrics is the causal cone.

The particular case $\mathcal{L}=e^{\frac{\psi}{2} F}$ with $\psi=\frac{3}{2} \gamma$ is similar to the previous Gauss-Bonnet theory. The hyperbolicity range coincides under the change $E \rightarrow H$ and $H \rightarrow E$.

\section{CONCLUSIONS}

Nonlinear generalization of Maxwell's theory arises naturally in many circumstances, some of which were 
discussed in the examples presented above. In most cases of interest, they are generated from a variational principle involving a Lagrangian function of the two Lorentz-invariant scalars one can form from Maxwell's tensor. It is well known that all these theories have a dispersion relation (set of hyperplanes allowing solutions which are constant along them) determined by the null vectors of two effective conformal Lorentzian metrics, which we call $\mathfrak{g}_{1}^{a b}$ and $\mathfrak{g}_{2}^{a b}$. These cones share a very important property: generically, they have a pair of null directions in common with the background metric, and thus a preferred two plane containing these two vectors is preferred, too [in some degenerate (nongeneric) cases, these two directions collapse into a single one]. Since they are null cones of conformal Lorentzian metrics, we can define their propagation cones, namely, the cone of all vectors of which norms with respect to each one of these metrics are positive definite. The shared null direction property implies that either the propagation cones are nested, one inside the other, or they are not, having no vector in common.

A first question one might ask when one is faced with any one of such generalizations is whether they are well posed, namely, whether the solutions are continuous functions of their initial data. Without this requirement, the theories are powerless; they do not have any predictive power. Using the covariant approach to symmetric hyperbolicity introduced by Geroch, we developed simple criteria for nonlinear electrodynamics theories arising from arbitrary Lagrangians to shield a well-posed system of evolution equations.

The criteria we found are of two kinds, and of course equivalent to each other. One of them is geometric in nature; it says that whenever the propagation cones of the two conformal effective metrics have a nonempty intersection the theories are symmetric hyperbolic. There are two cases here: in one of them, the conformal metrics propagation cones have also an intersection with that of the background relativistic metric, and in the other, this is not the case. As can be seen from the examples, the Born-Infeld model, Gauss-Bonnet model, Euler-Heisenberg model, and toy model II have cone intersection with the background metric, and only the toy model I does not. In this second case, the propagation has the particularity that the propagation is along spacelike directions with respect to the background metric; nevertheless, the theory is perfectly causal and has an initial value formulation in which data must be given along a timelike (with respect to the background metric) hypersurface. Of course, coupling this theory with other fields with causal propagation along the background metric would result in an ill-posed system. In addition, in both cases, one of the propagation cones is always inside the other, so causality is always given by the cone of the metric with the largest propagation cone, which, according to our definitions, is always $\mathfrak{g}_{a b}^{1}$. There are cases in which this propagation cone strictly contains the background metric cone, and so we have propagation speeds larger than "light," namely, larger than those allowed at the background propagation cone. In other cases, the smaller cone is strictly inside the background cone, and we have relativistic causality.

The other criteria are algebraic; the cones nonempty intersection is equivalent to the positivity of the scalar expression $\alpha_{1} \beta_{2}$ which can be explicitly computed from the Lagrangian function and its derivatives and depends only on the values of the two Lorentz-invariant quantities. Whether the biggest of the metrics propagation cones is contained or contains the background metric cone depends on whether the quantity $\gamma_{1}$ is bigger or smaller than the one for nondegenerate cases and on $b_{1}$ being negative or positive for degenerate cases. The algebraic criteria are very easy to check in actual examples. It is important to realize that whether a theory is hyperbolic or not might depend on the field strength of it (the values the invariant scalars take), and so along evolution, a perfectly nice solution might cease to be well posed. Using the algebraic conditions, one could characterize the set of theories in which hyperbolicity holds for all values of the field. That set of theories should be preferred. In addition, there are some generalizations to nonlinear electrodynamics, for instance, those that use non-Abelian fields (see, for instance, Ref. [60]); it would be nice to see how much of our analysis goes through in these cases.

The proof of the above-mentioned criteria involves the explicit construction of hyperbolizers; thus, when trying to evolve the equations of these theories, we provide a set of evolution equations which can be safely used. The propagation cones of these symmetrizers coincide with the smaller one of the effective metrics, and so we recuperate for them the same causality properties as discussed above. Thus, these hyperbolizations are optimal, and they allow for all possible hypersurfaces where initial data can be given.

We also assert that the constraints arising in the theories are in all cases integrable (in the sense of Geroch). In some sense, this is so because the constraint structure is close to the one of Maxwell. This means that in all cases, if they are satisfied for initial data and the system is hyperbolic, they remain satisfied along evolution as long as the system remains hyperbolic and inside the corresponding domain of dependence.

Notice that at no point do we use energy conditions; this is so because hyperbolicity depends on second-derivative conditions of the Lagrangian, while energy conditions involve only first derivatives. To some extent, energy conditions are related (through energy-momentum conservation) to causality conditions. Their imposition probably would prevent those cases in which the effective cones have no intersection with the space-time cone. But in those cases, one probably would require a different energy condition. 


\section{ACKNOWLEDGMENTS}

This work was supported by SeCyT-UNC Secretaría de Ciencia y Tecnología-Universidad Nacional de Córdoba, Córdoba, Argentina; CONICET Consejo Nacional de Investigaciones Científicas y Técnicas, Argentina; FONCyT Fondo para la Investigación Científica y Tecnológica, Argentina; MinCyT Ministerio de Ciencia, Tecnología e Innovación Productiva, Argentina and CAPES Foundation, Ministry of Education, Brasília/DF, Brazil. E. G. would like to thank CAPES-Brazil process 2383136 for financial support and DAMTP (Cambridge University), where part of this work was done.

\section{APPENDIX A: FRAMES}

To facilitate calculations, we work in a family of preferred frames; to build it, we use the principal null directions of $F_{a b}$. To display it, we use spinors [53-55], but this is not essential.

We know that in the spinorial form the electromagnetic field is

$$
F_{a b} \rightarrow \epsilon_{A B} \bar{\phi}_{A^{\prime} B^{\prime}}+\epsilon_{A^{\prime} B^{\prime}} \phi_{A B}
$$

with $\phi_{A B}=\phi_{(A B)}$ and

$$
\epsilon_{A B}=\left(\begin{array}{cc}
0 & 1 \\
-1 & 0
\end{array}\right)
$$

\section{Nondegenerate case}

In the nondegenerate case, $\phi_{A B}$ is characterized by two different principal null directions,

$$
\phi_{A B}=\phi \theta_{(A} \mu_{B)}
$$

where $\theta_{A} \mu^{A}=1$ and $\phi$ is a complex normalization factor. Using these spinors, we can construct a (complex) null tetrad,

$$
\begin{aligned}
k^{a} & \rightarrow \theta_{A} \bar{\theta}_{A^{\prime}} & l^{a} \rightarrow \mu_{A} \bar{\mu}_{A^{\prime}} \\
m^{a} & \rightarrow \theta_{A} \bar{\mu}_{A^{\prime}} & \bar{m}^{a} \rightarrow \bar{\theta}_{A^{\prime}} \mu_{A},
\end{aligned}
$$

where $k^{a} \eta_{a b} l^{b}=1, m^{a} \eta_{a b} \bar{m}^{a}=-1$, and all other contractions vanish.

We observe they are eigenvectors of the electromagnetic field, namely,

$$
\begin{aligned}
F^{a}{ }_{b} k^{b} & =\operatorname{Re}(\phi) k^{a} \quad F^{a}{ }_{b} l^{b}=-\operatorname{Re}(\phi) l^{a} \\
F^{a}{ }_{b} m^{b} & =i \operatorname{Im}(\phi) m^{a} \quad F^{a}{ }_{b} \bar{m}^{b}=-i \operatorname{Im}(\phi) \bar{m}^{a} .
\end{aligned}
$$

So, by noticing that $F=-\left(\phi^{2}+\bar{\phi}^{2}\right)$ and $G=i\left(\phi^{2}-\bar{\phi}^{2}\right)$, it follows that

$$
\begin{aligned}
& \operatorname{Re}(\phi)= \pm \sqrt{\frac{-F+\sqrt{F^{2}+G^{2}}}{4}} \\
& \operatorname{Im}(\phi)= \pm \sqrt{\frac{F+\sqrt{F^{2}+G^{2}}}{4}}
\end{aligned}
$$

Notice $G=\operatorname{Re}(\phi) \operatorname{Im}(\phi)$, and so the signs of $\operatorname{Re}(\phi)$ and $\operatorname{Im}(\phi)$ must be properly fixed according to the sign of $G$. Finally, we build our (real) tetrad as

$$
\begin{array}{rlrl}
\tau^{a} & =\frac{1}{\sqrt{2}}\left(k^{a}+l^{a}\right) & z^{a}=\frac{1}{\sqrt{2}}\left(k^{a}-l^{a}\right) \\
x^{a}=\frac{1}{\sqrt{2}}\left(m^{a}+\bar{m}^{a}\right) & y^{a}=\frac{i}{\sqrt{2}}\left(m^{a}-\bar{m}^{a}\right) .
\end{array}
$$

In this frame, the background metric is $\eta_{a b}=\operatorname{Diag}(1,-1,-1,-1) ; \tau^{a}$ is timelike, while $x^{a}, y^{a}, z^{a}$ are spacelike vectors. The electromagnetic field and its dual read

$$
\begin{aligned}
F_{i j}= & \left(\begin{array}{cccc}
0 & -E_{1} & -E_{2} & -E_{3} \\
E_{1} & 0 & H_{3} & -H_{2} \\
E_{2} & -H_{3} & 0 & H_{1} \\
E_{3} & H_{2} & -H_{1} & 0
\end{array}\right)=\left(\begin{array}{ccccc}
0 & 0 & 0 & -\operatorname{Re}(\phi) \\
0 & 0 & \operatorname{Im}(\phi) & 0 \\
0 & -\operatorname{Im}(\phi) & 0 & 0 \\
\operatorname{Re}(\phi) & 0 & 0 & 0
\end{array}\right) \\
F_{i j}^{*}=\left(\begin{array}{cccc}
0 & H_{1} & H_{2} & H_{3} \\
-H_{1} & 0 & E_{3} & -E_{2} \\
-H_{2} & -E_{3} & 0 & E_{1} \\
-H_{3} & E_{2} & -E_{1} & 0
\end{array}\right) & =\left(\begin{array}{cccc}
0 & 0 & 0 & \operatorname{Im}(\phi) \\
0 & 0 & \operatorname{Re}(\phi) & 0 \\
0 & -\operatorname{Re}(\phi) & 0 & 0 \\
-\operatorname{Im}(\phi) & 0 & 0 & 0
\end{array}\right),
\end{aligned}
$$

where it follows that $\vec{E}$ and $\vec{H}$ are parallel, both lying on the $z^{a}$ direction. And one can immediately see how calculations will become simpler by adopting this frame. 


\section{a. Boost freedom}

Since the length of the two principal null directions (or corresponding spinors) is arbitrary, there is a freedom on the frame choice which does not alter the above form of $F_{a b}$. If one considers the transformation $\theta_{A} \rightarrow \frac{1}{\varepsilon} \theta_{A}$ and $\mu^{A} \rightarrow \varepsilon \mu^{A}$, it can be noticed that $\phi_{A B}$, and therefore $F_{a b}$, remains unchanged. Thus, if we choose $\varepsilon$ real, the null tetrad (A2) changes to

$$
\begin{aligned}
\hat{k}^{a} & \rightarrow \frac{1}{\varepsilon^{2}} \theta_{A} \bar{\theta}_{A^{\prime}} & \hat{l}^{a} \rightarrow \varepsilon^{2} \mu_{A} \bar{\mu}_{A^{\prime}} \\
m^{a} \rightarrow \theta_{A} \bar{\mu}_{A^{\prime}} & \bar{m} & \rightarrow \bar{\theta}_{A^{\prime}} \mu_{A},
\end{aligned}
$$

and in the new frame,

$$
\begin{array}{rlrl}
\hat{\tau}^{a} & =\frac{1}{\sqrt{2}}\left(\hat{k}^{a}+\hat{l}^{a}\right) & \hat{z}^{a}=\frac{1}{\sqrt{2}}\left(\hat{k}^{a}-\hat{l}^{a}\right) \\
x^{a}=\frac{1}{\sqrt{2}}\left(m^{a}+\bar{m}^{a}\right) & y^{a}=\frac{i}{\sqrt{2}}\left(m^{a}-\bar{m}^{a}\right) .
\end{array}
$$

So, this freedom corresponds to a boost in the null plane defined by the two null directions. The choice of $\varepsilon$ imaginary causes a rotation of the frame components perpendicular to the null plane form by the two null directions. Both the metric $\eta_{a b}$ and Maxwell tensor components remain invariant.

\section{Degenerate case}

As before, the electromagnetic tensor takes the spinorial form (A1), but now there is just a single null direction associated to it:

$$
\phi_{A B}=\theta_{A} \theta_{B} .
$$

To complete the frame then, we shall choose an arbitrary null direction $\mu_{A}$, such that $\theta_{A} \mu^{A}=1$, and we proceed building a null tetrad like in (A5),

$$
\begin{aligned}
& k^{a} \rightarrow \frac{1}{\varepsilon^{2}} \theta_{A} \bar{\theta}_{A^{\prime}} l^{a} \rightarrow \varepsilon^{2} \mu_{A} \bar{\mu}_{A^{\prime}} \\
& m^{a} \rightarrow \theta_{A} \bar{\mu}_{A^{\prime}} \quad \bar{m} \rightarrow \bar{\theta}_{A^{\prime}} \mu_{A},
\end{aligned}
$$

where we set $k^{a} \eta_{a b} l^{b}=1, m^{a} \eta_{a b} \bar{m}^{a}=-1$, and in analogy to the previous case, any other contractions vanish, with $\varepsilon$ being a real positive parameter that we can freely pick up. ${ }^{12}$

It follows that

$$
\begin{aligned}
& F_{a b} k^{b}=0 ; \quad F_{a b} l^{b}=-\varepsilon^{2}\left(m_{a}+\bar{m}_{a}\right) ; \\
& F_{a b} m^{b}=-\varepsilon^{2} k_{a} ; \quad F_{a b} \bar{m}^{b}=-\varepsilon^{2} k_{a} .
\end{aligned}
$$

\footnotetext{
${ }^{12}$ Notice we strongly rely on this freedom in Sec. II C 2, particularly on proving Lemma 2.
}

Now, we build the frames as in (A3) and get the following expressions for $F_{a b}$ and $F_{a b}^{*}$ :

$$
\begin{aligned}
F_{i j} & =\left(\begin{array}{cccc}
0 & -E_{1} & -E_{2} & -E_{3} \\
E_{1} & 0 & H_{3} & -H_{2} \\
E_{2} & -H_{3} & 0 & H_{1} \\
E_{3} & H_{2} & -H_{1} & 0
\end{array}\right)=\left(\begin{array}{cccc}
0 & -\varepsilon^{2} & 0 & 0 \\
\varepsilon^{2} & 0 & 0 & -\varepsilon^{2} \\
0 & 0 & 0 & 0 \\
0 & \varepsilon^{2} & 0 & 0
\end{array}\right) \\
F_{i j}^{*} & =\left(\begin{array}{cccc}
0 & H_{1} & H_{2} & H_{3} \\
-H_{1} & 0 & E_{3} & -E_{2} \\
-H_{2} & -E_{3} & 0 & E_{1} \\
-H_{3} & E_{2} & -E_{1} & 0
\end{array}\right)=\left(\begin{array}{cccc}
0 & 0 & \varepsilon^{2} & 0 \\
0 & 0 & 0 & 0 \\
-\varepsilon^{2} & 0 & 0 & \varepsilon^{2} \\
0 & 0 & -\varepsilon^{2} & 0
\end{array}\right) .
\end{aligned}
$$

Thus, the vectors $\vec{E}=\left(\varepsilon^{2}, 0,0\right)$ and $\vec{H}=\left(0, \varepsilon^{2}, 0\right)$ are orthogonal each other (i.e., $G=0$ ) and have equal norms (i.e., $F=0$ ), as they should.

\section{APPENDIX B: DEGENERATE EFFECTIVE METRICS}

In this Appendix, we want to show that if one of the two effective metrics becomes degenerate (noninvertible) then the system is not symmetric hyperbolic. Such degenerate cases will occur whenever one of the variables in (16) becomes zero. From Eq. (18), we see that only the cases $\alpha_{1}=0$ or $\beta_{2}=0$ are allowed. When $\alpha_{1}=0$, say, the metric $g_{1}^{a b}$ will no longer be invertible. However, we stress here that the determinant from expression (41) still factorizes into four metrics $\left(\mathfrak{g}_{1}^{a b}, \mathfrak{g}_{2}^{a b}, \tilde{\mathfrak{g}}_{a b}^{1}, \mathfrak{g}_{a b}^{2}\right)$, where now $\tilde{\mathfrak{g}}_{a b}^{1} t^{a} t^{b}=$ $\beta_{1}\left(t_{0}^{2}-t_{3}^{2}\right)$ [Fig. 3a] is obviously not the inverse of $\mathfrak{g}_{1}^{a b}$. When $\beta_{2}=0$, the determinant factorizes into four metrics $\left(\mathfrak{g}_{1}^{a b}, \mathfrak{g}_{2}^{a b}, \mathfrak{g}_{a b}^{1}, \tilde{\mathfrak{g}}_{a b}^{2}\right)$, where $\tilde{\mathfrak{g}}_{a b}^{2} t^{a} t^{b}=-\alpha_{2}\left(t_{1}^{2}+t_{2}^{2}\right)$ [Fig. 3b].

We first analyze in detail the case $\alpha_{1}=0$. Suppose for contradiction there exist $n_{a}^{o}$ and $t_{o}^{b}$ such that the matrix $T_{\beta}^{\alpha}\left(n^{o}, t_{o}\right)$ has all its eigenvalues positive, and let us generically write $n_{a}^{o}=\left(n_{0}, n_{1}, n_{2}, n_{3}\right)$. Then, by following similar arguments to those used on the proofs of the main theorems, we will reach a contradiction. The construction

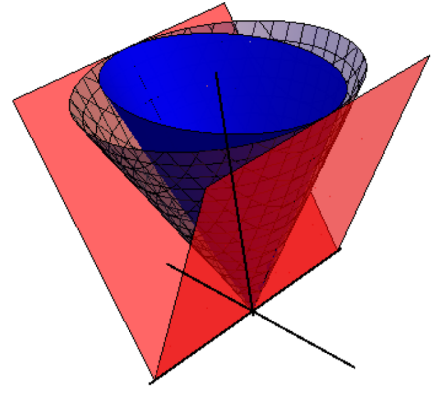

(a)

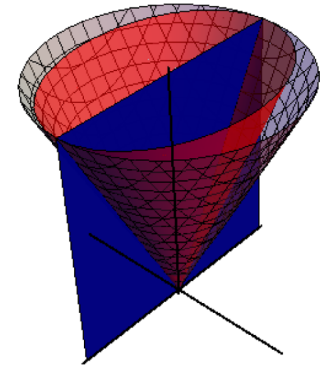

(b)
FIG. 3 (color online). Null surfaces of the metrics are illustrated: $\tilde{\mathfrak{g}}_{1 a b}$ (red), $\mathfrak{g}_{2 a b}$ (blue), and $\eta_{a b}$ (gray). (a) $\alpha_{1}=0$ and $\alpha_{2}, \beta_{1}, \beta_{2} \neq 0$ and (b) $\beta_{2}=0$ and $\alpha_{1}, \alpha_{2}, \beta_{1} \neq 0$. 
goes as follows. we first show it is possible to continuously connect $n_{a}^{o}$ with a second covector, $\hat{n}_{a}^{o}=\left(n_{0},-n_{1},-n_{2}, n_{3}\right)$, without changing along the path the sign of the eigenvalues. The path we propose is $\left(n_{0}, n_{1}, n_{2}, n_{3}\right) \rightarrow\left(n_{0}, \epsilon n_{1}\right.$, $\left.\epsilon n_{2}, n_{3}\right) \rightarrow\left(n_{0}, \epsilon n_{1}, 0, n_{3}\right) \rightarrow\left(n_{0}, \epsilon n_{1},-\epsilon n_{2}, n_{3}\right) \rightarrow\left(n_{0}, 0\right.$, $\left.-\epsilon n_{2}, n_{3}\right) \rightarrow\left(n_{0},-\epsilon n_{1},-\epsilon n_{2}, n_{3}\right) \rightarrow\left(n_{0},-n_{1},-n_{2}, n_{3}\right)$, for some positive but small parameter $\epsilon$.

Then, as argued at the beginning of Sec. IV, since both $H_{\alpha \beta}\left(n^{o}, t_{o}\right)$ and $H_{\alpha \beta}\left(\hat{n}^{o}, t_{o}\right)$ will be positive definite, it turns that $H_{\alpha \beta}\left(n^{o}+\hat{n}^{o}, t_{o}\right)$ must be positive definite as well. But a simple computation shows that $n_{a}^{o}+\hat{n}_{a}^{o}=2\left(n_{0}, 0,0, n_{3}\right)$, which should also be in the cone, is null for $\mathfrak{g}_{1}^{a b}$, and so one of the eigenvalues must be zero, leading us to a contradiction. Hence, to conclude, no such pair $\left(n_{a}^{o}, t_{o}^{b}\right)$ can exist.

The exact same construction (but now with $t^{a}$ ) can be applied when $\beta_{2}=0$, where one of the effective metrics in their covariant version degenerates. Thus, the system is not symmetric hyperbolic for any of these two "pathological" cases. However, the last case might still be strongly hyperbolic, and we shall study this case in a future work.
[1] H. Euler and B. Köckel, On the scattering of light from light in the Dirac theory, Naturwissenschaften 23, 246 (1935).

[2] W. Heisenberg and H. Euler, Consequences of Diracs theory of positrons, Z. Phys. 98, 714 (1936).

[3] R. Karplus and M. Neuman, The scattering of light by light, Phys. Rev. 83, 776 (1951).

[4] J. Schwinger, On gauge invariance and vacuum polarization, Phys. Rev. 82, 664 (1951).

[5] S. K. Blau, M. Visser, and A. Wipf, Analytic results for the effective action, Int. J. Mod. Phys. A 06, 5409 (1991).

[6] R. Soldati and L. Sorbo, Effective action for Dirac spinors in the presence of general uniform electromagnetic fields, Phys. Lett. B 426, 82 (1998).

[7] G. V. Dunne, The Heisenberg-Euler effective action: 75 years on, Int. J. Mod. Phys. Conf. Ser. 14, 42 (2012); Int. J. Mod. Phys. A27, 1260004 (2012).

[8] Y.-R. Shen, Principles of Nonlinear Optics (WileyInterscience, New York, NY, USA, 1984.

[9] M. Born and E. Wolf, Principles of Optics: Electromagnetic Theory of Propagation, Interference and Diffraction of Light (Cambridge University Press, Cambridge, England, 1999).

[10] N. Bretón, Nonlinear electrodynamics and cosmology, J. Phys. Conf. Ser. 229, 012006 (2010).

[11] V. A. De Lorenci, R. Klippert, M. Novello, and J. M. Salim, Nonlinear electrodynamics and FRW cosmology, Phys. Rev. D 65, 063501 (2002).

[12] M. Novello, S. E. Perez Bergliaffa, and J. Salim, Nonlinear electrodynamics and the acceleration of the universe, Phys. Rev. D 69, 127301 (2004).

[13] M. Novello, E. Goulart, J. M. Salim, and S. E. Perez Bergliaffa, Cosmological effects of nonlinear electrodynamics, Classical Quantum Gravity 24, 3021 (2007).

[14] R. García-Salcedo, T. Gonzalez, and I. Quiros, No compelling cosmological models come out of magnetic universes which are based on nonlinear electrodynamics, Phys. Rev. D 89, 084047 (2014).

[15] A. Montiel, N. Bretón, and V. Salzano, Parameter estimation of a nonlinear magnetic universe from observations, Gen. Relativ. Gravit. 46, 1758 (2014).

[16] A. D.García, H. I. Salazar, and J. F. Plebański et al., Type- $D$ solutions of the Einstein and Born-Infeld nonlinear-electrodynamics equations, Il Nuovo Cimento B 84, 65 (1984).

[17] N. Bretón, Born-Infeld black hole in the isolated horizon framework, Phys. Rev. D 67, 124004 (2003).

[18] W. A. Chemissany, M. De Roo, and S. Panda, Thermodynamics of Born-Infeld black holes, Classical Quantum Gravity 25, 225009 (2008).

[19] S. Gunasekaran, D. Kubizňák, and R. B Mann, Extended phase space thermodynamics for charged and rotating black holes and Born-Infeld vacuum polarization, J. High Energy Phys. 11 (2012) 110.

[20] E. Ayon-Beato and A. Garcia, Regular Black Hole in General Relativity Coupled to Nonlinear Electrodynamics, Phys. Rev. Lett. 80, 5056 (1998).

[21] N. Bretón and S. Esteban Perez Bergliaffa, On the thermodynamical stability of black holes in nonlinear electrodynamics, Ann. Phys. (Amsterdam) 354, 440 (2015).

[22] M. Born and L. Infeld, Foundations of the new field theory, Nature (London) 132, 1004 (1933).

[23] M. Born, Modified field equations with a finite radius of the electron, Nature (London) 132, 282 (1933).

[24] M. Born and L. Infeld, Foundations of the new field theory, Proc. R. Soc. A 144, 425 (1934).

[25] G. W. Gibbons, Aspects of Born-Infeld theory and string/ m-theory, Rev. Mex. Fis. 49S1, 19 (2003).

[26] R. Geroch, Partial differential equations of physics, Gen. Relativ. Gravit., 19 (1996).

[27] R. Courant and D. Hilbert, Methods of Mathematical Physics, (Cambridge University Press Archive, Cambridge, 1966), Vol. 2.

[28] P. D. Lax, Hyperbolic PartialDifferential Equations (Courant Institute of Mathematical Sciences, New York, 2006).

[29] L. Hörmander, Lectures on Nonlinear Hyperbolic Differential Equations (Springer Science and Business Media, Berlin Heidelberg, 1997), vol. 26.

[30] Y. Brenier, Hydrodynamic structure of the augmented Born-Infeld equations, Arch. Ration. Mech. Anal. 172, 65 (2004).

[31] P. D. Lax, Hyperbolic Systems of Conservation Laws and the Mathematical Theory of Shock Waves (SIAM, Philadelphia, 1973), Vol. 11. 
[32] A. Majda, Compressible Fluid Flow and Systems of Conservation Laws in Several Space Variables (Springer Science and Business Media, New York, 2012), Vol. 53.

[33] D. Serre, Systems of Conservation Laws 1: Hyperbolicity, Entropies, Shock Waves (Cambridge University Press, Cambridge, England, 1999).

[34] D. Serre, Hyperbolicity of the nonlinear models of Maxwells equations, Arch. Ration. Mech. Anal. 172, 309 (2004).

[35] C. M. Dafermos, Hyperbolic conservation laws in continuum physics, volume 325 of grundlehren der mathematischen wissenschaften [fundamental principles of mathematical sciences] (Springer-Verlag, Berlin, 2010).

[36] S. Demoulini, D. M. A. Stuart, and A. E. Tzavaras, A variational approximation scheme three-dimensional elastodynamics with polyconvex energy, Arch. Ration. Mech. Anal. 157, 325 (2001).

[37] B. D. Coleman and E. H. Dill, Thermodynamic restrictions on the constitutive equations of electromagnetic theory, Z. Angew Math. Phys. 22, 691 (1971).

[38] V. Perlick, On the hyperbolicity of Maxwell's equations with a local constitutive law, J. Math. Phys. (N.Y.) 52, 042903 (2011).

[39] J. Speck, The nonlinear stability of the trivial solution to the Maxwell-Born-Infeld system, J. Math. Phys. (N.Y.) 53, 083703 (2012).

[40] D. Christodoulou, The Action Principle and Partial Differential Equations (Princeton University, Princeton, NJ, 2000), vol. 146.

[41] D. Christodoulou and S. Klainerman, Asymptotic properties of linear field equations in minkowski space, Commun. Pure Appl. Math. 43, 137 (1990).

[42] M. Novello, M. Visser, and G. Volovik, Artificial Black Holes (World Scientific, Singapore, 2002).

[43] C. Barceló, S. Liberati, and M. Visser, Analogue gravity, Living Rev. Relativity 8, 214 (2005).

[44] G. Boillat, Nonlinear electrodynamics: Lagrangians and equations of motion, J. Math. Phys. (N.Y.) 11, 941 (1970).

[45] J. F. Plebanski, Lectures on Non-Linear Electrodynamics (Nordita, Sweden, 1970).

[46] J. Hadamard, Leçons sur la propagation des ondes et les équations de l'hydrodynamique, par Jacques Hadamard (University of Michigan Library, Michigan, 1903).
[47] Y. N. Obukhov and G. F. Rubilar, Fresnel analysis of wave propagation in nonlinear electrodynamics, Phys. Rev. D 66, 024042 (2002).

[48] Y. N. Obukhov, T. Fukui, and G. F. Rubilar, Wave propagation in linear electrodynamics, Phys. Rev. D 62, 044050 (2000).

[49] G. F. Rubilar, Y. N. Obukhov, and F. W. Hehl, Generally covariant Fresnel equation and the emergence of the light cone structure in linear pre-metric electrodynamics, Int. J. Mod. Phys. D 11, 1227 (2002).

[50] M. Novello, V. A. De Lorenci, J. M. Salim, and R. Klippert, Geometrical aspects of light propagation in nonlinear electrodynamics, Phys. Rev. D 61, 045001 (2000).

[51] V. A. De Lorenci, R. Klippert, M. Novello, and J. M. Salim, Light propagation in non-linear electrodynamics, Phys. Lett. B 482, 134 (2000).

[52] É. Goulart de Oliveira Costa and S. Esteban Perez Bergliaffa, A classification of the effective metric in nonlinear electrodynamics, Classical Quantum Gravity 26, 135015 (2009).

[53] R. Penrose and W. Rindler, Spinor and Twistor Methods in Space-Time Geometry, Spinors and Space-Times (Cambridge University Press, Cambridge, England, 1988), vol. 2.

[54] F. A. E. Pirani, Introduction to gravitational radiation theory, Lect. Gen. Relativ. 1, 249 (1964).

[55] R. M. Wald, General Relativity (University of Chicago, Chicago, 2010).

[56] K. Hoffman and R. A. Kunze, Linear Algebra (PrenticeHall, Englewood Cliffs, 1971).

[57] G. W. Gibbons and C. A. R. Herdeiro, Born-Infeld theory and stringy causality, Phys. Rev. D 63, 064006 (2001).

[58] B. Zwiebach, Curvature squared terms and string theories, Phys. Lett. 156B, 315 (1985).

[59] H. Kleinert, E. Strobel, and S. S. Xue, Fractional effective action at strong electromagnetic fields, Phys. Rev. D 88, 025049 (2013).

[60] O. Obregon, Non-Abelian Born-Infeld theory without the square-root, Mod. Phys. Lett. A 21, 1249 (2006). 